\title{
Refinement properties for relational structures
}

C. C. Chang (Los Angeles, Calif.), B. Jónsson (Minneapolis, Min.), A. Tarski* (Berkeley, Calif.)

1. Introduction. By a relational structure or, more briefly, a structure, we shall here mean a system $\mathfrak{U}=\left\langle A, R_{i, t \in T}\right.$ consisting of a non-empty set $A$ and a family of finitary relations $R_{t}$, indexed by a set $T$. (The precise meaning of most terms used in this introduction will be explained later.) The restriction to finitary relations is not essential. In case each of the relations $R_{t}$ is a function, i.e., an operation (for instance, each ternary relation is a binary operation), we refer to $\mathfrak{A}$ as an algebraic structure or, simply, as an algebra. One of the most important notions in the general theory of relational structures is that of direct, or cardinal, product, a familiar concept from modern algebra. The operation of direct multiplication when applied to two similar structures $\mathfrak{B}$ and $\mathfrak{C}$, or to a family of similar structures $\mathfrak{B}_{i}$ indexed by a set $I$, yields a new similar structure

$$
\mathfrak{B} \times \mathfrak{C}, \quad \text { or } \quad \mathbf{P}_{i \in I} \mathfrak{B}_{i}
$$

respectively. This operation is applied for two purposes. On the one hand we can use it to construct complicated structures with prescribed properties from given ones. On the other hand we can use it to study properties of a complicated structure $\mathfrak{A}$ by representing it isomorphically as a direct preduct of simpler structures $\mathfrak{B}_{i}$, symbolically

$$
\mathfrak{U} \cong \mathbf{P}_{i \in I} \mathfrak{B}_{i} .
$$

This formula is said to give a direct decomposition of $\mathfrak{A}$ into factors $\mathfrak{B}_{i}$.

In this paper we shall discuss the notion of cardinal product primarily from the second point of view. ( $\left.{ }^{1}\right)$ In this connection the notion of a (di-

* This paper was prepared for publication while the anthors were engaged in research projects supported by U. S. National Science Foundation grants number G-14006, G-17957 and G-14092.

(1) Most of the results in this paper were first stated without proofs in Chang [2] and in Jónsson and Tarski [7] and [9]. A detailed presentation of some of Chang's contributions ean be found in his doctoral dissertation, Cardinal and ordinal factorization of relation types, which was prepared under the guidance of Tarski and submitted to the University of California, Berkeley, 1955. 
rectly) indecomposable structure is especially relevant. A structure $\mathfrak{U}=\left\langle A, R_{t}\right\rangle_{t \in T}$ is said to be indecomposable if it has more than one element and if in every direct decomposition of $\mathfrak{A}$ into two factors, $\mathfrak{A} \cong \mathfrak{B} \times \mathfrak{C}$, one of the factors $\mathfrak{B}$ and $\mathfrak{C}$ consists of just one element. (If none of the relations $R_{t}$ is empty, then this implies that the other factor is isomorphic to $\mathfrak{A}$.) One is particularly interested in knowing whether. or not a given structure $\mathfrak{A}$ can be represented as a direct product of indecomposable structures, and whether this representation is unique up to isomorphism. More precisely, we ask whether or not $\mathfrak{A}$ has the following two properties:

(E) There exist indecomposable structures $\mathfrak{B}_{i}$, associated with the elements $i$ of some set $I$, such that

$$
\mathfrak{A} \cong \mathbf{P}_{i \in I} \mathfrak{B}_{i}
$$

(U) If (1) holds, and if also

$$
\mathfrak{U} \cong \mathbf{P}_{j \in J} \widetilde{C}_{j}
$$

where all the structures $B_{i}$ with $i \in I$ and $C_{j}$ with $j \in J$ are indecomposable, then there exists a one-to-one map $\varphi$ of $I$ onto $J$ such that $\mathfrak{B}_{i} \cong \mathfrak{C}_{p(i)}$ for all $i \in I$. To illustrate the importance of these properties, consider a class $K$ of structures satisfying the following conditions: (i) together with any structure $\mathfrak{A}$ it contains all isomorphic images of $\mathfrak{A}$; (ii) together with any structures $\mathfrak{B}_{i}$ it contains their direct product, and conversely; (iii) every structure $\mathfrak{A}$ in $\boldsymbol{K}$ has the properties (E) and (U). Under these conditions, using familiar arguments we can solve a series of fundamenta. problems concerning structures in the class $\boldsymbol{K}$. For instance, we can show that, for any structures $\mathfrak{A}, \mathfrak{B}, \mathfrak{C}$ in $\boldsymbol{K}$, (iv) the formula $\mathfrak{A} \times \mathfrak{A} \cong$ $\cong \mathfrak{B} \times \mathfrak{B}$ implies $\mathfrak{U} \cong \mathfrak{B}$, and $(v)$ the formula $\mathfrak{U} \times \mathfrak{B} \times \mathfrak{C} \cong \mathfrak{A}$ implies $\mathfrak{A} \times \mathfrak{B} \cong \mathfrak{U} \times \mathfrak{C} \cong \mathfrak{U}$. $\left(^{2}\right)$ On the other hand, a solution (either positive or negative) of the same problems for classes $\boldsymbol{K}$ some of whose members fail to satisfy either (E) or (U) frequently presents considerable diffieulty. $\left({ }^{3}\right)$

Obviously every finite structure has the property (E), and for algebras it is easy to formulate various chain conditions that imply the property (E). Apart from such finiteness conditions, no general and useful criteria are known that permit us to show for comprehensive classes $\boldsymbol{K}$ of structures that the property (E) applies to each member of $\boldsymbol{K}$. Of course, if (E) fails, then (U) is trivially satisfied, but is of no

(2) Under the assumptions (i)-(iii) the algebra of isomorphism types of structures in $\boldsymbol{K}$ under direct multiplication is isomorphic to a direct power of the algebra of cardinal numbers under addition. Compare analogous remarks in Tarski [10], pp. $250 \mathrm{f}$.

( $\left.{ }^{3}\right)$ See Chang [3], where further bibliographical references are given. interest. This is perhaps the main reason why the property (U) is frequently replaced in discussion by another property that implies (U) and that is of considerable interest in its application to arbitrary structures independent of whether or not they are decomposable into indecomposable factors. We have in mind the following refinement property:

$(\mathrm{R})$ If (1) and (2) are any decompositions of $\mathfrak{A}$ (not necessarily into indecomposable factors), then there are structures $\mathfrak{D}_{i, j}$ such that

$$
\mathfrak{B}_{p} \cong P_{j \in J} \mathfrak{D}_{p, j} \quad \text { and } \quad \mathfrak{C}_{q} \cong \mathbf{P}_{i \in I} \mathfrak{D}_{i, q}
$$

for all $p \in I$ and $q \in J$.

Obviously every relational structure with the property $(R)$ also has the property $(\mathrm{U})$. The converse is not true, since there exist structures that satisfy neither (E) nor (R), and for such a structure (U) is trivially satisfied. It is more interesting to observe that apparently it is not even the case that $(\mathrm{E})$ and $(\mathrm{U})$ jointly imply $(\mathrm{R})$. We use the word "apparently" because the only known counter-example makes use of the following very special case of the Generalized Continuum Hypothesis: There exists an infinite cardinal $m$ such that for every cardinal $n$ the condition $2^{m}=2^{n}$ implies that $m=n$. Assuming that $m$ has this property, let $\mathfrak{A}$ be a Boolean group of order $2^{n}$. Then

$$
\mathfrak{A} \cong \mathbf{P}_{i \in I} \mathfrak{B}_{i}
$$

where the order of $I$ is $m$ and, for each $i \epsilon I, \mathfrak{B}_{i}$ is a 2 -element group. The groups $\mathfrak{B}_{i}$ are indecomposable, and from our assumption concerning $m$ it follows that this representation of $\mathfrak{A}$ as a direct product of indecomposable structures is unique up to isomorphism. On the other hand,

$$
\mathfrak{U} \cong \mathfrak{B} \times \mathfrak{U}
$$

where $\mathfrak{B}$ is a countable Boolean group. For any system of 2-element groups $\mathfrak{C}_{j}, j \in J$, the direct product

$$
\mathbf{P}_{j \in J} \widetilde{C}_{j}
$$

is either finite or non-denumerable, and is therefore not isomorphic to $\mathfrak{B}$. From this it follows that the two representations of $\mathfrak{A}$ do not have isomorphic refinements.

It is an essential feature of the above example that, although the structure $\mathfrak{U}$ has the property $(\mathrm{E})$, the direct factor $\mathfrak{B}$ of $\mathfrak{A}$ does not have this property. This can be seen from the following theorem, whose proof is quite easy.

For every structure $\mathfrak{A}$, the conditions $(\alpha)$ and $(\beta)$ are equivalent:

$(\alpha)$ I has the properties ( $\mathrm{R})$ and $(\mathrm{E})$.

$(\beta) \mathfrak{A}$ has the property (U), and every direct factor of $\mathfrak{A}$ has the property (E). 
It is well known that in case $\mathfrak{A}$ is an algebra each of the factors $\mathfrak{B}_{i}$ in the decomposition (1) can be replaced by an algebra of the special form $\mathfrak{U} / F_{i}$ where $F_{i}$ is a congruence relation over $\mathfrak{A}$. Conversely, for suitable systems of congruence relations $F_{i}, i \in I$, we obtain a decomposition of $\mathfrak{A}$,

$$
\mathfrak{U} \cong \mathbf{P}_{i \in I} \mathfrak{A} / F_{\imath} \cdot\left(^{4}\right)
$$

Thus (3) represents so to speak a standard decomposition of $\mathfrak{A}$. In the present paper we extend the notion of a standard decomposition to arbitrary relational structures. More generally, we obtain standard decompositions for a quotient structure $\mathfrak{A} / E$,

$$
\mathfrak{U} / E \cong \mathbf{P}_{i \in I} \mathfrak{X} / F_{i},
$$

and this gives rise to the concept of a direct product

$$
E=\prod_{i \in I} F_{i}
$$

of a system of equivalence relations over $A$.

Concentrating on standard decompositions we obtain stronger forms of the properties (U) and (R). Thus $\mathfrak{A}$ is said to have the strict unique decomposition property if there exists, apart from the order of the factors, just one standard decomposition (3) such that all the quotients $\mathfrak{A} / F_{\text {, }}$ are indecomposable. If is said to have the strict refinement property if for any two standard decompositions

$$
\mathfrak{U} \cong \mathbf{P}_{i \in I} \mathfrak{A} / F_{i} \quad \text { and } \quad \mathfrak{A} \cong \mathbf{P}_{j \in \mathcal{J}} \mathfrak{A} / G_{j}
$$

there exist equivalence relations $H_{i, j}$ yielding standard decompositions

$$
\mathfrak{U} / F_{p} \cong \mathbf{P}_{j \in J} \mathfrak{U} / H_{p, j}, \quad \mathfrak{A} / G_{q} \cong \mathbf{P}_{i \in I} \mathfrak{I} / H_{i, q}
$$

for all $p \in I$ and $q \in J$. It is obvious that this implies that $\mathfrak{Q}$ has the refinement property.

It will be shown that the strict refinement property is equivalent to certain commutativity and distributivity conditions involving the relations $F_{i}$ and $G_{j}$. By somewhat relaxing these conditions we arrive at the so-called intermediate refinement property, which together with some mild additional conditions also implies the refinement property $(R)$. These two properties, the strict refinement property and the intermediate

(4) For the case of a finite set $I$, necessary and sufficient conditions on the congruence relations $F_{i}$ are given in Birkhoff [1], p. 87. (As has been observed by several writers, there is a minor inaccuracy in the formulation of these conditions: It must be assumed that each $F_{i}$ commutes with the intersection $\bigcap_{i \in \mathcal{J}} F_{i}$ for every non-empty index sets can be found in Hashimoto $[6]$, p. 96 .
ins all $j \in I$.) An extension to infinite refinement property, are the main topics of this paper. The notation and terminology is described in Section 2 , and Section 3 contains some preliminary results on direct products of equivalence relations. In Sections 4 and 5 we present our principal results on the strict refinement property, and Section 6 contains parallel results for the intermediate refinement property. Sections 7 and 8 contain applications to various classes of structures. The results given there include as special cases a number of known results concerning the refinement property, for instance for lattice ordered structures and for centerless algebras. (For a survey of such results and for bibliographical references, see [3].)

2. Terminology and notation. In addition to more standard set-theoretic notation that needs no explanation, we write $\mathbf{P}_{i \in I} A_{i}$ for the Cartesian product of the sets $A_{i}$ with $i \in I$, and ${ }^{I} A$ for the set of all functions on $I$ into $A$. Identifying each natural number $n$ with the set $\{0,1, \ldots, n-1\}$ of all natural numbers $k<n$, we write in particular ${ }^{n} A$ for the set of all $n$-termed sequences $x=\left\langle x_{0}, x_{1}, \ldots, x_{n-1}\right\rangle$ whose terms belong to $A$. By an $n$-ary relation we mean a set of $n$-termed sequences. By the field of an $n$-ary relation $F$ we mean the set of all elements that are terms of sequences that belong to $F$. An $n$-ary relation $F$ is said to be reflexire if $\langle u, u, \ldots, u\rangle \in F$ whenever $u$ belongs to the field of $F$. If $F$ is a binary relation we usually write $x F y$ for $\langle x, y\rangle \in F$. If $F$ and $G$ are binary relations, then by their relative product, $F \mid G$, we mean the set of all ordered pairs $\langle x, y\rangle$ such that $x F z G y$ for some element $z$, and by the converse of $F, \breve{F}$, we mean the set of all ordered pairs $\langle x, y\rangle$ such that $y F x$. A binary relation $F$ is said to be symmetric if $\widetilde{F}=F$, antisymmetric if the conditions $x F y$ and $y F x$ jointly imply that $x=y$, and transitive if $F \mid F C F$. By an equivalence relation we mean a binary relation that is symmetric and transitive. An equivalence relation whose fieldis $A$ is called an equivalence relation over $A$. Among the equivalence relations over $A$ are the identity relation, id $A_{A}$, consisting of all ordered pairs $\langle x, \infty\rangle$ with $x \in A$, and the universal relation, ${ }^{2} A$, consisting of all ordered pairs $\langle x, y\rangle$ with $x, y \in A$.

We shall have occasions to use various elementary facts from the calculus of binary relations, such as the associativity and monotonicity of the operation $\mid$, and the modular laws: For any binary relations $F, G$ and $H$,

$$
\begin{array}{lll}
\breve{F} \mid H \subseteq H & \text { implies } & (F \mid G) \cap H \subseteq F \mid(G \cap H) \\
H \mid \breve{F} \subseteq H & \text { implies } & H \cap(G \mid F) \subseteq(H \frown G) \mid F .
\end{array}
$$

We shall also make use of the fact that if $F$ and $G$ are equivalence relations over the same set $A$, then the conditions $F|G \subseteq G| F$ and $F|G=G| F$ are equivalent, and are necessary and sufficient for $F \mid G$ to be an equivalence 
relation over $A$. We further recall that if a family of of equivalence relations over a set $A$ is closed under the operations $\mid$ and $\cap$ (which, accordin to the last remark, implies that $F|G=G| F$ for all $F, G \in \mathcal{A}$ ), then $\mathcal{A}$ is a modular lattice under these operations.

A function whose values are positive integers is called a similarity type. Given a similarity type $\varrho$ with domain $T$, by a $\varrho$-structure we mean a system $\mathfrak{A}=\left\langle A, R_{t}\right\rangle_{t \in T}$ such that $A$ is a non-empty set and $R_{t} \subset^{e(t)} A$ for $t \in T$. In working with isomorphisms $f$ between $\varrho$-structures and with other maps of one $\varrho$-structure into another one, or into itself, we often have to consider the image under $f$ of a finite sequence $x=\left\langle x_{0}, x_{1}, \ldots, x_{n-1}\right\rangle$. For notational simplicity we take advantage of the fact that the image of $x$ under $f$ is simply the composition of the functions $f$ and $x,-$ in symbols $f x=f \circ x=\left\langle f\left(x_{0}\right), f\left(x_{1}\right), \ldots, f\left(x_{n-1}\right)\right\rangle$. Thus the definition of isomorphism can be formulated as follows: We say that $f$ is an isomorphism of the $\varrho$-structure $\mathfrak{U}=\left\langle A, R_{t}\right\rangle_{t \in T}$ onto the $\varrho$-structure $\mathfrak{B}=\left\langle B, S_{t}\right\rangle_{t \in T},-$ in symbols $f: \mathfrak{A} \cong \mathfrak{B}$, - if and only if $f$ is a one-to-one map of $A$ onto $B$ and, for all $t \in T$ and $x \epsilon^{e(t)} A$, the conditions $x \in R_{t}$ and $f \circ x \in S_{t}$ are equivalent.

Three methods for constructing new $\varrho$-structures from given ones will be needed: substructures, direct products, and quotient structures. If $\mathfrak{U}=\left\langle A, R_{t}\right\rangle_{\epsilon \in T}$ is a $\varrho$-structure and $B$ is a non-empty subset of $A$, then by the restriction of $\mathfrak{A}$ to $B$ - in symbols $\mathfrak{A}(B)$ - we mean the $\varrho$-structure $\left\langle B, R_{t} \cap{ }^{e(t)} B\right\rangle_{t \in T}$. If with each element $i$ of a non-empty set $I$ there is associated a $\varrho$-structure $\mathfrak{U}_{i}=\left\langle A_{i}, R_{i, t}\right\rangle_{t \in T}$, then by their direct product - in symbols $\mathbf{P}_{i \in I} \mathfrak{A}_{i}$ - we mean the $\varrho$-structure $\mathfrak{B}=\left\langle B, S_{t}\right\rangle_{t \in T}$ such that $B=\mathbf{P}_{i \in T} A_{i}$ and, for each $t \in T, S_{t}$ is the set of all $x \epsilon^{e(t)} B$ such that $\left\langle x_{0}(i), x_{1}(i), \ldots, x_{e(t)-1}(i)\right\rangle \in R_{i, t}$ for all $i \in I$.

Before discussing the concept of a quotient structure we introduce a useful notational convention. Suppose that $E$ is a binary relation and $A$ is the field of $E$. If $x \in A$, then by $x / E$ we mean the set of all elements $y \in A$ such that $x E y$. If $X$ is a subset of $A$, then by $X / E$ we mean the family of all sets $x / E$ associated with elements $x$ of $X$. There is a natural way of extending this notation to various members of the hierarchy built up from $A$. Thus, if $f$ is a function on a set $I$ into $A$, then by $f / E$ we mean the function $g$ on $I$ into $A / E$ such that $g(i)=f(i) / E$ for all $i \epsilon I$. Observe in particular that if $x$ is an $n$-termed sequence whose terms belong to $A$, then

$$
x / E=\left\langle x_{0} / E, x_{1} / E, \ldots, x_{n-1} / E\right\rangle
$$

is an $n$-termed sequence whose terms beong to $A / E$. If $R$ is an $n$-ary relation whose field is contained in $A$, then by $R / E$ we mean the $n$-ary relation consisting of all $n$-termed sequences $x / E$ associated with $n$-termed sequences $x$ that belong to $R$. Finally, if $\mathfrak{A}=\left\langle A, R_{t}\right\rangle_{t \in T}$ is a $\varrho$-structure, then by the quotient structure of $\mathfrak{U} \bmod E$-in symbols $\mathfrak{X} / E$ - we mean the $\varrho$-structure $\left\langle A\left|\mathbb{E}, R_{t}\right| \mathbb{E}\right\rangle_{t \in T}$.

It should be noted that in what follows we could restrict ourselves to considering quotient structures $\mathfrak{Q} / \mathbb{E}$ for certain special equivalence relations $E$, the so-called factor relations of $\mathfrak{A}$ defined in the next section. In case $\mathfrak{A}$ is an algebra it turns out that the factor relations of $\mathfrak{A}$ form a subset of the set of all congruence relations over $\mathfrak{A}$, and therefore the corresponding quotient structures are themselves algebras.

3. Factor relations. Throughout this paper we assume that $\varrho$ is a similarity type with domain $T$, and that $\mathfrak{A}=\left\langle A, R_{t ; t \in T}\right.$ is a $\varrho$-structure with the property that each of the relations $R_{t}$ is non-empty. Structures that do not satisfy this condition obviously do not have the refinement property; this follows from the observation that if $\mathfrak{U}$ is isomorphic to a direct product of $\varrho$-structures $\mathfrak{B}_{i}=\left\langle B_{i}, S_{i, t, t \in T}\right.$, then $R_{\imath}$ is empty if and only if one of the relations $S_{i, t}$ is empty.

We begin this section by describing the process for associating with any decomposition of a quotient $\mathfrak{A} / E$ a standard decomposition of $\mathfrak{T} / E$. This leads to the definitions of a direct product of equivalence relations over $A$ and of a factor relation. These concepts are characterized intrinsically, and some elementary properties are listed.

Theorem 3.1. Suppose $f: \mathfrak{U} / E \cong \mathbf{P}_{i \in I} \mathfrak{B}_{i}$ where $E$ is an equivalence relation over $A, I$ is a non-empty set, and for each $i \in I, \mathfrak{B}_{i}$ is a $\varrho$-structure. For $i \in I$ let

$$
F_{i}=\left\{\langle x, y\rangle \mid x, y \in A \text { and }(f(x / E))_{i}=(f(y / E))_{i}\right\} .
$$

Thein

(i) $E=\cap\left\{F_{i} \mid i \in I\right\}$. $x \in A$.

(ii) For each $i \in I, \quad g_{i}: \mathfrak{A} / F_{i} \cong \mathfrak{B}_{i}$ where $g_{i}\left(x / F_{i}\right)=(f(x / E))_{i}$ for all

(iii) $h: \mathfrak{U} / E \cong \mathbf{P}_{i \in I} \mathfrak{Q} / F_{i}$ where $(h(x / E))_{i}=x \mid F_{i}$ for all $x \in A$ and $i \in I$. Proof. For $i \in I$ let $\mathfrak{B}_{i}=\left\langle B_{i}, S_{i, t}\right\rangle_{t \in \boldsymbol{T}}$, and let

$$
\mathbb{C}=\left\langle C, U_{i}\right\rangle=\mathbf{P}_{i \in I} \mathfrak{B}_{i}, \quad \mathbb{D}=\left\langle D, V_{i}\right\rangle_{\epsilon \in T}=\mathbf{P}_{i \in I} \mathfrak{A} / F_{i} .
$$

Clearly $E$ is contained in each of the equivalence relations $F_{i}$. On the other hand if, for all $i \in I, x F_{i} y$ and hence $(f(x / E))_{i}=(f(y / E))_{i}$, then $f(x / E)=f(y / E)$, and since $f$ is one-to-one this implies that $x / E=y / E$ or, equivalently, $x E y$. Thus (i) holds.

For each $i \in I$ the fact that $E \subset F_{i}$ implies that the function $g_{i}$ on $A / F_{i}$ into $B_{i}$ can be so defined that $g_{i}\left(x / F_{i}\right)=(f(x / E))_{i}$ for all $x \in A$. That $g_{i}$ is one-to-one follows easily from the definition of $F_{i}$. Furthermore, given $b \in B_{i}$ there exists $c \in C$ such that $c_{i}=b$, and choosing $x \in A$ so that $f(x / E)=c$ we infer that $g_{i}(x / E)=b$. Thus $g_{i}$ maps $A / F_{i}$ onto $B_{i}$. Consider 
finally an element $t \in T$ and a sequence $X \epsilon^{e(t)}\left(A / F_{i}\right)$. If $X \in R_{t} / F_{i}$, then $X=x / F_{i}$ for some $x \in R_{t}$, hence $x / E \in R_{t} / E, f_{\circ}(x / E) \in U_{t}$, so that the sequence

$$
g_{i} \circ X=\left\langle\left(f\left(x_{0} / E\right)\right)_{i},\left(f\left(x_{1} / E\right)\right)_{i}, \ldots,\left(f\left(x_{e(t)-1} / E\right)\right)_{i}\right\rangle
$$

belongs to $S_{i, t}$. Conversely, if $g_{i} \circ X \in S_{i, t}$, and if we choose $x \in{ }^{o(t)} A$ so that $X=x / F_{i}$, then (1) holds. For $j \in I$ with $j \neq i$ choose $u^{j} \in S_{j, t}^{\prime}$, let $u^{i}$ be the sequence $g_{i} \circ X$, and let $c$ be the member of ${ }^{2(t)} C$ such that $c_{k}(j)=u_{i}^{i}$ for all $j \in I$ and $k<\varrho(t)$. Then $o \in U_{t}$, so that $c=f \circ(y \mid E)$ for some $y \in R_{t}$. It follows that $y / F_{i} \in R_{t} / F_{i}$, but $\left(f\left(y_{k} / E\right)\right)_{i}=c_{k}(i)=u_{k}^{i}=g_{i}\left(X_{k}\right)$ $=\left(f\left(x_{k} / E\right)\right)_{i}$, and therefore $y / F_{i}=x / F_{i}=X$. Consequently $X \in R_{t} / F_{i}$. Thus each $g_{i}$ is an isomorphism.

The isomorphisms $g_{i}$ in (ii) give rise to an isomorphism $h^{\prime}$ of $\mathbb{C}$ onto $\mathfrak{D}$ such that, for all $c \in C$ and $i \in I,\left(h^{\prime}(c)\right)_{i}=g_{i}^{-1}\left(c_{i}\right)$. To prove (iii) we need ouly verify that, for all $x \in A$,

$$
\left(h^{\prime} \circ f(x / E)\right)_{i}=g_{i}^{-1}\left((f(x / E))_{i}\right)=g_{i}^{-1}\left(g_{i}\left(x / F_{i}\right)\right)=x / F_{i} .
$$

DEFINITION 3.2. (i) Suppose that $I$ is a non-empty set and, for each $i \in I, F_{i}$ is an equivalence relation over $A$. Let $E=\bigcap\left\{F_{i} \mid i \in I\right\}$. We say that $E$ is the direct product of the relations $F_{i}-i n$ symbols

$$
E=\prod_{i \in I} F_{i}
$$

if $h: \mathfrak{a} / E \cong \mathbf{P}_{i \in I} \mathfrak{U} / F_{i}$ where $(h(x / E))_{i}=x / F_{i}$ for all $x \in A$ and $i \in I$.

(ii) We let

$$
\prod_{i \in \boldsymbol{g}} F_{i}={ }^{2} A .
$$

(iii) If $G$ and $H$ are equivalence relations over $A$, then we let

$$
G \times H=\prod_{i \in 2} F_{i}
$$

where $F_{0}=G$ and $F_{1}=H$ - provided this product exists.

DEFINITION 3.3. (i) Suppose that $E$ is an equivalence relation over $A$. $B y$ a factor relation of $E$ over $\mathfrak{A}$ we mean an equivalence relation $F$ over $A$ such that $E=F \times F^{\prime}$ for some equivalence relation $F^{\prime}$ over $A$. We let $F R(\mathfrak{H}, E)$ be the set of all factor relations of $E$ over $\mathfrak{N}$.

(ii) By a factor relation of $\mathfrak{A}$ we mean a factor relation of $i d_{1}$ over $\mathfrak{A}$. We let $F R(\mathfrak{I})=F R\left(\mathfrak{A}, i d_{A}\right)$.

THEOREM 3.t. Suppose that $I$ is a non-empty set, $F_{t}$ is an equivalence relation over $A$ for each $i \in I$, and $E=\bigcap\left\{F_{i} \mid i \in I\right\}$. Then

$$
E=\prod_{i \in I} F_{i}
$$

if and only if the following conditions hold:

(i) For each $x \in{ }^{I} A$. there exists $u \in A$ such that $x_{i} F_{i} u$ for all $i \in I$.

(ii) For all $t \in T$ and $x \epsilon^{e(t)} A$, if $x / F_{i} \in R_{t} / F_{i}$ for all $i \in I$, then $x / E \in R_{t} / E$.

Proof. Let $\mathfrak{D}=\left\langle D, V_{t}\right\rangle_{t \in T}=\mathbf{P}_{i \epsilon I} \mathfrak{U} / F_{i}$, and let $h$ be the function on $A / E$ into $D$ such that $(h(x / E))_{i}=x / F_{i}$ for all $x \in A$ and $i \in I$.

Suppose that $E$ is the direct product of the relations $F_{i}$. Then, by $3.2(\mathrm{i}), h: \mathfrak{A} / E \cong \mathfrak{D}$. Given $x \in{ }^{I} A$, let $X$ be the member of $D$ such that $X_{i}=x_{i} / F_{i}$ for all $i \in I$. Then there exists $u \in A$ such that $X=h(u / E)$, and hence for every $i \in I, u / F_{i}=(h(u / E))_{i}=X_{i}=x_{i} / F_{i}, x_{i} F_{i} u$. Under the hypothesis of (ii) the sequence $X=h \circ(x / E)$ belongs to $V_{t}$ because $\left\langle X_{0}(i), X_{1}(i), \ldots, X_{e(t)-1}(i)\right\rangle=x / F_{i} \in R_{t} / F_{i}$ for all $i \in I$. Consequently $x / E \in R_{t} / E$. Thus (i) and (ii) are necessary.

Suppose that (i) and (ii) hold. From the definition of $E$ it readily follows that $h$ is a one-to-one map of $A / E$ into $D$. Given $X \in D$, there exists $x \epsilon^{I} A$ such that $X_{i}=x_{i} / F_{i}$ for all $i \epsilon I$. By (i) there exists $u \in A$ such that, for each $i \in I, x_{i} F_{i} u$, and hence $X_{i}=u / F_{i}$. Consequently $X=h(u / E)$. Thus $h$ maps $A / E$ onto $D$.

Consider any $t \in T$ and $X \epsilon^{e(t)}(A / E)$. If $X \in R_{t} / E$, then there exists $x \in R_{t}$ such that $X=x / E$, hence $x / F_{i} \in R_{t} / F_{i}$ for all $i \in I$, and consequently $h \circ X=h(x / E) \in V_{t}$. Conversely, if $h \circ X \in V_{t}$, and if we choose $x \in A$ so that $X=x / E$, then for each $i \in I$ we have $\left(x / F_{i}\right)_{k}=\left(h\left(x_{k} \mid E\right)\right)_{i}$ for $k=0,1, \ldots, \varrho(t)-1$, and therefore $x / F_{i} \in R_{t} / F_{i}$. Consequently, by (ii), $X=x / E \in R_{t} / E$. This shows that $h$ is an isomorphism, and $E$ is therefore the direct product of the relations $F_{t}$.

We now list some simple properties of the operation of direct multiplication of equivalence relations over $A$. The letters $E, F, G$ and $H$, with or without subscripts, are assumed to denote equivalence relations over $A$, and whenever a statement asserts that two direct products are equal, this is to be understood to include the assertion that both products exist provided one of them does.

Corollary 3.5. (i) If $\prod_{i \in I} F_{i}$ exists and $J \subset I$, then $\prod_{i \in J} F_{i}$ exists.

(ii) If $J$ is the union of pairwise disjoint sets $J_{t}$ with $i \in I$, then $\prod_{i \in I} \prod_{j \in J_{i}} F_{j}=\prod_{j \in J} F_{j}$.

(iii) If $\varphi$ is a one-to-one map of $J$ onto $I$, then $\prod_{i \in I} F_{i}=\prod_{j \in J} F_{\varphi(j)}$.

(iv) If $I \cap J=\emptyset$, then $\prod_{i \in I} F_{i} \times \prod_{i \in J} F_{i}=\prod_{i \in I \cup J} F_{i}$.

(v) $\prod_{i \in I} \prod_{j \in J} F_{t, j}=\prod_{j \in J} \prod_{i \in I} F_{i, j}$. 
(vi) If $E=\prod_{i \in I} F_{i}=\prod_{i \in I} G_{i}$, and if $F_{i} \subseteq \underline{G} G_{i}$ for all $i \in I$, then $F_{i}=G_{i}$ for all $i \in I$.

(vii) If $\prod_{i \in J \cup K} F_{i}$ exists, then $\left(\prod_{i \in J} F_{i}\right) \mid\left(\prod_{i \in K} F_{i}\right)=\prod_{i \in, J \cap K} F_{i}$.

Proof. Under the hypothesis of (i), if $x \epsilon^{J} A$, we can find $y \epsilon^{I} A$ that agrees with $x$ on $J$, and by 3.4 (i) there exists $u \in A$ such that $y_{i} F_{i} u$ for all $i \in I$ and hence $x_{i} F_{i} u$ for all $i \in J$. Let $E=\cap\left\{F_{i} \mid i \in I\right\}$ and $E^{\prime}=\bigcap\left\{F_{i} \mid i \in J\right\}$. Consider any $t \in T$, and choose $y \in R_{t}$. If $x \epsilon^{e(t)} A$,

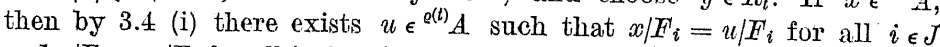
and $y / F_{i}=u / F_{i}$ for all $i \in I-J$. If $x / F_{i} \in R_{t} / F_{i}$ for all $i \in J$, then $u / F_{i} \in R_{t} / F_{i}$ for all $i \in I$, hence $u / E \in R_{t} / \mathbb{E}$ by 3.4 (ii). Since $E \subseteq E^{\prime}$, this implies that the sequence $x / E^{\prime}=u / E^{\prime}$ belongs to $R_{t} / E^{\prime}$. Thus (i) holds.

We first prove (ii) under the hypothesis that the direct product

$$
E=\prod_{j \in J} F_{j}
$$

exists, whence it follows by (i) that the direct products

$$
G_{i}=\prod_{j \in J_{i}} F_{j}
$$

exist for all $i \in I$. If $x \epsilon^{I} A$, then by 3.4 (i) there exists $u \in A$ such that $x_{i} F_{j} u$ whenever $i \in I$ and $j \in J_{i}$, and hence $x_{i} G_{i} u$ for all $i \in I$. Furthermore, if $t \in T$ and $x \epsilon^{o(t)} A$ are such that $x / G_{i} \in R_{t} / G_{i}$ for all $i \in I$, then $x / F_{j} \in R_{t} / F_{j}$ for all $j \in J$, and it follows by 3.4 (ii) that $x / E \in R_{t} / E$. Consequently

$$
E=\prod_{i \in I} G_{i}
$$

Assume now that the products (2) and (3) exist. If $x \epsilon^{J} \mathcal{A}$, we apply 3.4 (i) twice to infer, first that there exists $y \in{ }^{I} A$ such that $x_{j} F_{j} y_{i}$ whenever $i \in I$ and $j \in J_{i}$, and second that there exists $u \in A$ such that $y_{i} G_{i} u$ for all $i \in I$. Since $G_{i} \subseteq F_{j}$ whenever $i \in I$ and $j \in J_{i}$, we see by the transitivity of the relations $F_{j}$ that $x_{j} F_{j} u$ for all $j \in J$. Furthermore, if $t \in T$ and $x \epsilon^{e^{(l)}} A$ are such that $x / F_{j} \in R_{t} / F_{j}$ for all $j \in J$, then we apply 3.4 (ii) twice to infer that $x / G_{i} \in R_{t} / G_{i}$ for all $i \in I$, and hence that $x / E \in R_{t} / E$. Thus (1) holds.

The statement (iii) is obvious, and (iv) and ( $v$ ) are immediate consequences of (ii). Under the hypothesis of (vi), if $p \in I$ and $x G_{p} y$, then by 3.4 (i) we can choose $u \in A$ so that $x F_{p} u$ and $y F_{i} u$ whenever $p \neq i \in I$. Then $y G_{i} u$ for all $i \in I$, and we infer successively that $y E u, y F_{p} u, x F_{p} y$. Thus $F_{p}=G_{p}$.

Finally, if the hypothesis of (vii) is satisfied, then the three direct products involved in the conclusion exist by (i). If $x F_{i} y$ for all $i \in J \cap K$, then by 3.4 (i) we may choose $u \in A$ such that $x F_{i} u$ for all $i \epsilon J$ and $y F_{i} u$ for all $i \in K-J$. It follows that $y F_{i} u$ for all $i \in K$, and therefore

$$
x\left(\prod_{i \in J} F_{i}\right) u \quad \text { and } \quad u\left(\prod_{i \in \mathbb{K}} F_{i}\right) y .
$$

Thus

$$
\prod_{i \in J \cap K} F_{i} \subseteq\left(\prod_{i \in J} F_{i}\right) \mid\left(\prod_{i \in K} F_{i}\right)
$$

The opposite inclusion is obvious.

Corollary 3.6. (i) $F \times G=G \times F$.

(ii) $F \times(G \times H)=(F \times G) \times H$.

Proof: by 3.5 (iii), (ii).

COROLlaRr 3.7. $F \times G$ exists if and only if $F \mid G={ }^{2} A$ and, for all $t \in T$ and $x \epsilon^{e(t)} A$, the conditions $x / F \in R_{t} / F$ and $x / G \in R_{t} / G$ jointly imply that $x /(F \cap G) \epsilon R_{t} /(F \cap G)$.

Proof: by 3.4 and 3.2 (iii).

CoRoLlary 3.8. (i) If $F^{\prime} \in F R(\mathfrak{A}, E)$, then $F R(\mathfrak{A}, F) \subseteq F R(\mathfrak{A}, E)$.

(ii) If $E=\prod_{i \in I} F_{i}$, then $F_{i} \in F R(\mathfrak{A}, E)$ for all $i \in I$.

Proof: by 3.6 (ii) and 3.5 (iv).

4. Refinement properties. After giving a precise definition of the term "refinement property" which has already been employed informally, we introduce and investigate the stronger concept of the strict refinement property. The principal result of this section, Theorem 4.5 , gives several necessary and sufficient conditions in order for $\mathfrak{A}$ to have this latter property.

DEFINITION 4.1. We say that $\mathfrak{A}$ has the refinement property if and only if, given any $\varrho$-structures $\mathfrak{B}_{i}$ and $\mathfrak{C}_{j}$ associated with all the elements $i$ of $a$ set $I$ and $j$ of a set $J$, the conditions

$$
\mathfrak{U} \cong P_{i \in I} \mathfrak{B}_{i} \quad \text { and } \quad \mathfrak{A} \cong P_{j \in J} \mathfrak{C}_{j}
$$

jointly imply that there exist $\varrho$-structures $\mathcal{D}_{i, j}$ associated with all the elements $i$ of $I$ and $j$ of $J$ such that, for all $p \in I$ and $q \in J$,

$$
\mathfrak{B}_{p} \cong P_{j \in J} \mathfrak{D}_{p, j} \quad \text { and } \quad \mathfrak{C}_{q} \cong P_{i \in I} \mathfrak{D}_{i, q} .
$$

DEFINITION 4.2. Given an equivalence relation $E$ over $A$, we say that $(\mathfrak{A}, E)$ has the strict refinement property if and only if, for any equivalence relations $F_{i}$ and $G_{j}$ over $A$, associated with all the elements $i$ of $a$ set $I$ and $j$ of a set $J$, the conditions

$$
E=\prod_{i \in I} F_{i} \quad \text { and } \quad E=\prod_{j \in J} G_{j}
$$


jointly imply that there exist equivalence relations $H_{i, j}$ associated with all the elements $i$ of $I$ and $j$ of $J$, such that for all $p \in I$ and $q \in J$,

$$
F_{p}=\prod_{j \in J} H_{p, j} \quad \text { and } \quad G_{q}=\prod_{i \in I} H_{i, q}
$$

Using Theorem 3.1 we obtain:

COROLLARY 4.3. If $E$ is an equivalence relation over $A$, and if (2r, $E$ ) has the strict refinement property, then $\mathfrak{U} / E$ has the refinement property.

It is not difficult to show that whether or not ( $\mathfrak{A}, E$ ) has the strict refinement property depends only on the isomorphism type of the $\varrho$-structure $\mathfrak{A} / E$. However, we shall not give a direct proof of this fact, since it will be obtained later, in Corollary 4.6, as an immediate consequence of the main theorem of this section.

LEMMA 4.4. Suppose that $E$ is an equivalence relation over $A$ and, for all $i \in I$ and $j \in J, F_{i}, G_{j}$ and $H_{i, j}$ are equivalence relations over $A$. If

$$
E=\prod_{i \in I} F_{i}=\prod_{j \in J} G_{j}
$$

and if, for all $p \in I$ and $q \in J$,

$$
F_{p}=\prod_{j \in J} H_{p, j} \quad \text { and } \quad G_{q}=\prod_{i \in I} H_{i, q}
$$

then $H_{i, j}=F_{i}\left|G_{j}=G_{j}\right| F_{i}$ for all $i \in I$ and $j \in J$.

Proof. Consider elements $p \in I$ and $q \in J$ and let $P$ be the set of all ordered pairs $\langle p, j\rangle$ with $j \in J$ and $Q$ the set of all ordered pairs $\langle i, q\rangle$ with $i \in I$. Then $P \cap Q=\{\langle p, q\rangle\}$ and using 3.5 (i)-(iii) to show that the direct product

$$
\prod_{\langle i, j\rangle \in P \cup Q} H_{i, j}
$$

exists, we infer by 3.5 (vii) that

$$
F_{p}\left|G_{q}=\left(\prod_{\langle i, j\rangle \in P} H_{i, j}\right)\right|\left(\prod_{\langle i, j\rangle \in Q} H_{i, j}\right)=\prod_{\langle i, j\rangle \in P \cap Q} H_{i j}=H_{u, q} .
$$

THEOREM 4.5. If $E$ is an equivalence relation over $A$, then the following conditions are equivalent:

(i) $(\mathfrak{A}, E)$ has the strict refinement property.

(ii) For all $F, F^{\prime}, G, G^{\prime} \in F R(\mathfrak{A}, E)$, if $E=F \times F^{\prime}=G \times G^{\prime}$, then there exist $H_{0}, H_{1}, H_{2}, H_{3} \in F R(\mathfrak{A}, E)$ such that $F=H_{0} \times H_{1}, F^{\prime}=H_{2} \times H_{3}$, $G=H_{0} \times H_{2}$ and $G^{\prime}=H_{1} \times H_{3}$.

(iii) $F R(\mathfrak{A}, E)$ is a Boolean algebra under the operations $\mid$ and $\cap$. (iv) For all $F, F^{\prime}, G \in F R(\mathfrak{A}, E)$, if $E=F \times F^{\prime}$, then $F|G=G| F$ and $(F \mid G) \cap F^{\prime} \underline{C} G$.

(v) Fror all $F^{\prime}, F^{\prime}, G \in F R(\mathfrak{A}, E)$, if $E=F \times F^{\prime}$, then $(F|G| F) \cap F^{\prime} \subseteq G$.

(vi) For all $F, F^{\prime}, G \in F R(\mathfrak{A}, E)$, if $E=F \times F^{\prime}$, then $G \subseteq F^{\prime}\left(F^{\prime} \cap G\right)$ C. $\left(F^{\prime} \cap G\right) \mid F$.

Proof. Clearly (i) implies (ii). Conversely, assume that (ii) holds, and suppose

$$
E=\prod_{i \in I} F_{i}=\prod_{j \in J} G_{j} .
$$

For $p \in I$ and $q \in J$, if we let

then by 3.5 (iv)

$$
\bar{F}_{p}=\prod_{p \neq i \in I} F_{i}, \quad \bar{G}_{q}=\prod_{q \neq j \in J} G_{j},
$$

$$
E=F_{p} \times \bar{F}_{p}=G_{q} \times \bar{G}_{q},
$$

and it follows by 4.4 that

$$
G_{q}=\left(F_{p} \mid G_{q}\right) \times\left(\bar{F}_{p} \mid G_{q}\right) .
$$

Consequently, and by $3.5(\mathrm{v})$,

$$
\begin{aligned}
E & =\prod_{q \in J}\left(\left(F_{p} \mid G_{q}\right) \times\left(\bar{F}_{p} \mid G_{q}\right)\right) \\
& =\prod_{q \in J}\left(F_{p} \mid G_{q}\right) \times \prod_{q \in J}\left(\bar{F}_{p} \mid G_{q}\right) .
\end{aligned}
$$

Since in this last product the first factor contains $F_{p}$ and the second factor contains $\bar{F}_{p}$, we see by 3.5 (vi) that

$$
F_{p}=\prod_{q \in J}\left(F_{p} \mid G_{q}\right)
$$

Since this holds for every $p \in I$ and, similarly,

$$
G_{q}=\prod_{p \in I}\left(F_{p} \mid G_{q}\right)
$$

for all $q \in J$, we conclude that (i) is satisfied.

We next show that the last three statements are equivalent. With $F, F^{\prime}$ and $G$ as given there, if (iv) holds, then

$$
\begin{aligned}
\left(F^{\prime} \cap G\right) \mid F & \supseteq\left(F^{\prime} \cap\left(F^{\prime} \mid G\right) \cap F^{\prime}\right)\left|F=\left((F \mid G) \cap F^{\prime}\right)\right| F \\
& =\left(F^{\prime} \mid G\right) \cap\left(F^{\prime} \mid F^{\prime}\right)=F|G=G| F^{\prime}
\end{aligned}
$$

Consequently $\left(F^{\prime} \cap G\right)|F=G| F \supseteq G$. Recalling that the relative product of two equivalence relations over $A$ is an equivalence relation over $A$ 
if and only if they commute, we infer further that $\left(F^{\prime} \cap G\right)|F=F|\left(F^{\prime} \cap G\right)$. Thus (vi) holds.

If (vi) holds, then

$$
\begin{aligned}
& F|G=F|\left(F^{\prime} \cap G\right)=\left(F^{\prime} \cap G\right)\left|F^{\prime}=G\right| F^{\prime}, \\
& (F|G| F) \cap F^{\prime}=(F \mid G) \cap F^{\prime}=\left(F \mid\left(F^{\prime} \cap G\right)\right) \cap F^{\prime} \\
& =\left(F^{\prime} \cap F^{\prime}\right) \mid\left(F^{\prime} \cap G\right)=F^{\prime} \cap G \subseteq G,
\end{aligned}
$$

yielding the conclusion of $(v)$. Finally, if $(v)$ holds, then

$$
F|G \supseteq F|\left(\left(F|G| F^{\prime}\right) \cap F^{\prime}\right)=\left(F^{\prime} \mid F^{\prime}\right) \frown(F|G| F)=F|G| F^{\prime} \supseteq G \mid F,
$$

so that $F|G=G| F$, and

$$
(F \mid G) \frown F^{\prime} \subseteq(F|G| F) \cap F^{\prime} \subseteq G,
$$

proving (iv). The three statements are therefore equivalent.

If (ii) holds, and if $F, F^{\prime}$ and $G$ are as in (iv), then it follows from 4.4 that $F^{\prime}|G=G| F^{\prime}$ and $G=(F \mid G) \cap\left(F^{\prime} \mid G\right) \supseteq(F \mid G) \frown F^{\prime}$. Hence (iv) holds. Conversely, suppose (iv) is satisfied. If $E=F \times F^{\prime}=G \times G^{\prime}$, then

$$
\begin{gathered}
(F \mid G) \cap\left(F \mid G^{\prime}\right)=F \mid\left(G \cap\left(F \mid G^{\prime}\right)\right)=F, \\
(F \mid G)\left|\left(F \mid G^{\prime}\right) \supseteq G\right| G^{\prime}={ }^{2} A .
\end{gathered}
$$

Furthermore, if $t \in T$ and $x \epsilon^{\mathrm{o}(t)} A$ are such that $x /(F \mid G) \in R_{t} /(F \mid G)$ and $x /\left(F \mid G^{\prime}\right) \in R_{t} /\left(F / G^{\prime}\right)$, then there exist $y, z \in R_{t}$ such that $x /(F \mid G)=y /(F \mid G)$ and $x /\left(F \mid G^{\prime}\right)=z /\left(F^{\prime} \mid G^{\prime}\right)$. We can then find $u \epsilon^{e^{(t)}} A$ such that $y / G=u / G$ and $z / G^{\prime}=u / G^{\prime}$. Thus $u / G \in R_{t} / G$ and $u / G^{\prime} \in R_{t} / G^{\prime}$, whence it follows that $u / E \in R_{t} / E$ or, in other words, that $u / E=v / E$ for some $v \in R_{t}$. Consequently $x /(F \mid G)=v /(F \mid G)$ and $x /\left(F \mid G^{\prime}\right)=v /\left(F \mid G^{\prime}\right)$, hence $x / F=v / F$ $\epsilon R_{t} / F$. We have thus proved that $F=(F \mid G) \times\left(F \mid G^{\prime}\right)$. This shows that (ii) holds.

Since (iii) obviously implies (iv), it only remains to show that (ii) implies (iii). Assuming (ii), we infer by 4.4 that $F|G=G| F \in F R(\mathfrak{A}, E)$ for all $F, G \in F R(\mathfrak{A}, E)$, and that if $E=F \times F^{\prime}=G \times G^{\prime}$, then $F \cap G$ $=\left(F^{\prime} \mid G\right) \cap\left(F \mid G^{\prime}\right) \cap\left(F^{\prime} \mid G\right)$. Since $E$ is the direct product of the four factor relations $(F \mid G),\left(F \mid G^{\prime}\right),\left(F^{\prime} \mid G\right)$ and $\left(F^{\prime} \mid G^{\prime}\right)$, it follows that the direct product of the first three of these relations exists and is equal to $F \cap G$, and hence that $F \cap G$ is a factor relation of $E$. Thus $F R(\mathfrak{U}, E)$ is closed under the operations $\mid$ and $\cap$, and is therefore a complemented modular lattice under these operations. To prove that this lattice is distributive, and hence a Boolean algebra, it sufficies to show that if $F, F^{\prime}$ and $G$ are any members of the lattice, and if $E=F^{\prime} \times F^{\prime}$, then $G=(F \cap G) \mid\left(F^{\prime} \cap G\right)$. Since (ii) implies (iv) and hence also (vi), we have $G \subseteq F \mid\left(G \cap F^{\prime}\right)$, and the desired equation therefore follows by the modular law.
The proof of the theorem is now complete.

COROLIARY 4.6. Suppose that $E$ is an equivalence relation over $A$. Then $(\mathfrak{H}, E)$ has the strict refinement property if and only if $\mathfrak{H} / E$ has the strict refinement property.

Proof. The correspondence $F \rightarrow \bar{F}=F / E$ is an isomorphism of the lattice $\mathcal{L}$ of all those equivalence relations over $A$ that contain $E$ onto the lattice $\overline{\mathfrak{L}}$ of all equivalence relations over $A / E$. It is easy to verify that this correspondence maps $F R(\mathfrak{A}, E)$ onto $F R(\mathfrak{U} / E)$ and that, for all $F, G$ in $\mathcal{L}$, the conditions $F|G=G| F$ and $\bar{F}|\bar{G}=\bar{G}| \bar{F}$ are equivalent. Consequently, $F R(\mathfrak{A}, E)$ is a Boolean algebra under the operations and $n$ if and only if $F R(\mathfrak{X} / E)$ is a Boolean algebra under these operations. From this the corollary readily follows by 4.5 .

5. Decomposition functions. The notion of a decomposition function will serve roughly the same role as the projections associated with a direct decomposition of an algebra with a zero element. We could associate a system of decomposition functions with any representation of $i d_{.1}$ as a dircet product of factor relations, but it is simpler to consider only direct products of two factors, and because of Theorem 4.5 this turns out to be sufficient.

Definition 5.1. By a decomposition function over $\mathfrak{A}$ we mean a function $f$ on ${ }^{2} A$ into $A$ with the following properties:

(i) For all $x \in A, f(x, x)=x$.

(ii) For all $x, y, z \in A, f(f(x, y), z)=f(x, z)=f(x, f(y, z))$.

(iii) For all $t \in T$ and $x, y, z \epsilon^{\varrho(t)} A$, if $x, y \in R_{t}$ and $z_{k}=f\left(x_{k}, y_{k}\right)$ whenever $k<\varrho(t)$, then $\approx \in R_{t}$.

We let. $D F(\mathfrak{A})$ be the set of all decomposition functions over $\mathfrak{A}$.

If $f$ is a function on ${ }^{2} A$ into $A$, then we let $f^{\text {a }}$ be the function on ${ }^{2} A$ into $A$ such that $f^{a}(x, y)=f(y, x)$ for all $x, y \in A$, and for each $u \in A$ we let $f_{u}$ be the function on $A$ into $A$ such that $f_{u}(x)=f(x, u)$ for all $x \in A$.

Corollary 5.2. Suppose that $F, F^{\prime} \in F R(\mathfrak{A})$ and $i d_{-1}=F \times F^{\prime}$, and let $f$ be the unique function on ${ }^{2} A$ into $A$ such that, for all $x, y \in A$,

Then $f \in D F(\mathfrak{X})$ and, for any element $u \in A$,

$$
\begin{aligned}
F & =\left\{\langle x, y\rangle \mid x, y \in A \text { and } f_{u}(x)=f_{u}(y)\right\}, \\
F^{\prime} & =\left\{\langle x, y\rangle \mid x, y \in A \text { and } f_{u}^{d}(x)=f_{u}^{a}(y)\right\} .
\end{aligned}
$$

Conversely, if $f \in D F(\mathfrak{A})$ and $u \in A$, and if $F$ and $F^{\prime}$ are defined by (ii) and (iii), respectively, then $i d_{A}=F \times F^{\prime}$ and (i) holds.

Proof. Under the hypothesis of the first part, $f$ obviously satisfies the first condition of 5.1. Also, for any $x, y, z \in A, x F f(x, y)$ and 
$f(x, y) F f(f(x, y), z)$, hence $x F f(f(x, y), z)$. Furthermore, replacing $x$ and $y$ by $f(x, y)$ and $z$ in (i) we find that $f(f(x, y), z) F^{\prime} z$. Since $f(x, z)$ is the unique element $v$ such that $x F v$ and $v F^{\prime} z$, we infer that the first equality in 5.1 (ii) holds. The second equality is proved similarly.

Finally, if $x, y, z$ satisfy the conditions of 5.1 (iii), then $z / F=x / F$ $\epsilon R_{t} \mid F^{\prime}$ and $z / F^{\prime}=y\left|F^{\prime} \epsilon R_{t}\right| F^{\prime}$, and since $F \cap F^{\prime}=i d_{A}$, we infer by 3.7 that $z \in R_{t}$. Thus $f \in D F(\mathfrak{U})$.

For any elements $x, y, u \in A$ we have $x H f_{u}(x)$ and $y F f_{u}(y)$. Hence if $f_{u}(x)=f_{u}(y)$, then $x F y$. Conversely, if $x F y$, then by (i) $f_{u}(x) F f_{u}(y)$. But $f_{u}(x) F^{\prime} u$ and $f_{u}(y) F^{\prime} u$, hence $f_{u}(x) F^{\prime} f_{u}(y)$, and since $F \cap F^{\prime}=i d_{A}$, we infer that $f_{u}(x)=f_{u}(y)$. Thus (ii) holds, and (iii) can be proved similarly.

Now suppose $f \in D F(\mathfrak{H})$ and $u \in A$, and let $F$ and $F^{\prime}$ be defined by (ii) and (iii). Then $F^{\prime}$ and $F^{\prime}$ are equivalence relations over $A$. It follows from 5.1 (ii) that (i) holds for all $x, y \in A$, and hence that $F \mid F^{\prime}={ }^{2} A$. Furthermore, if $x F y$ and $x F^{\prime} y$, then

$$
x=f(f(x, u), f(u, x))=f(f(y, u), f(u, y))=y .
$$

Hence $F \cap F^{\prime}=i d_{A}$. Finally, if $t \in T$ and $z \epsilon^{{ }^{e(t)}} A$ are such that $z / F^{\prime} \in R_{t} / F$ and $z / F^{\prime} \in R_{t} / F^{\prime \prime}$, then there exist $x, y \in R_{t}$ such that $z / F=x / F$ and $z / F^{\prime}=y \mid F^{\prime}$. Then for each $k<\varrho(t)$ we have $x_{k} F z_{k}$ and $z_{k} F^{\prime} y_{k}$, hence $z_{k}=f\left(x_{k}, y_{k}\right)$. According to 5.1 (iii) this implies that $z \in R_{t}$. We have thus shown that $i d_{A}=F^{\prime} \times F^{\prime}$.

DeFINITION 5.3. If $i d_{A}=F \times F^{\prime}$, then by the decomposition function associated with $F$ and $F^{\prime}$ we mean the unique member $f$ of $D F(\mathfrak{H})$ such that, for all $x, y \in A, x F f(x, y)$ and $f(x, y) \mathbb{H}^{\prime} y$.

COROLLARY 5.4. If $i d_{A}=F \times F^{\prime}$, and if $f$ is the decomposition function associated with $F$ and $F^{\prime \prime}$, then $f^{d}$ is the decomposition function associated with $F^{\prime}$ and $F$.

LEMOA 5.5. If $f \in D F(\mathfrak{A})$ and $x, y, u, v, w \in A$, then the following statements hold:
(i) $f_{u}(u)=u$.
(ii) $f_{v} f_{u}(x)=f_{v}(x)$.
(iii) $f^{d}{ }_{u} f_{v}(x)=f^{d}{ }_{u}(v)$.
(iv) $f_{u}(x)=x$ if and only if $f^{d}{ }_{u}(x)=u$.
(v) $f_{u}(x)=f_{v}(y)$ implies that $f_{w}(x)=f_{w}(y)$.
(vi) $f_{u}(x)=f_{u}(y)$ if and only if $f_{v}(x)=f_{v}(y)$.
(vii) $f_{u}(x)=f_{u}(y)$ and $f^{d}{ }_{u}(x)=f^{d}{ }_{u}(y)$ if and only if $x=y$.

Proof. The first statement is just another form of $5.1(\mathrm{j})$, and from 5.1 (ii) we infer that $f_{v} f_{u}(x)=f(f(x, u), v)=f(x, v)=f_{v}(x)$ and $f_{u}^{d} f_{v}(x)=f(u, f(x, v))=f(u, v)=f^{a}{ }_{u}(v)$. Hence (ii) and (iii) hold. By (iii) and (i), if $f_{u}(x)=x$, then $f^{d}{ }_{u}(x)=f^{d}{ }_{u} f_{u}(x)=u$. Conversely, if $f^{d}{ }_{u}(x)=u$, then by 5.1 (ii), (i), $f_{u}(x)=f(x, u)=f\left(x, f^{d} u(x)\right)=f(x, f(u, x))=f(x, x)=x$. This proves (iv).

Using (ii) we see that the condition $f_{u}(x)=f_{v}(y)$ implies that $f_{w}(x)$ $=f_{w} f_{u}(x)=f_{w} f_{v}(y)=f_{w}(y)$. Thus (v) holds, and hence so does the special case (vi). Finally, if $f_{u}(x)=f_{u}(y)$ and $f^{d}{ }_{u}(x)=f_{u}^{d}(y)$, then by 5.1 (i), (ii), $x=f(x, x)=f(f(x, u), f(u, x))=f\left(f_{u}(x), f^{d} u(x)\right)=f\left(f_{u}(y), f^{d}{ }_{u}(y)\right)=f(y, y)$ $=y$. Oonsequently (vii) is also satisfied.

THEOREM 5.6. The following conditions are equivalent:

(i) $\mathfrak{A}$ has the strict refinement property.

(ii) For all $f, g \in D F(\mathfrak{H})$ and $u \in A, f_{u} g_{u}=g_{u} f_{u}$.

(iii) Given any $f, g \in D F(\mathfrak{H})$ and $x_{0}, x_{1}, x_{2}, x_{3} \in A$, there exists $u \in A$ such that $f_{u} g_{u}\left(x_{i}\right)=g_{u} f_{u}\left(x_{i}\right)$ and $f^{d}{ }_{u} g_{u}\left(x_{i}\right)=g_{u} f^{d}{ }_{u}\left(x_{i}\right)$ for $i=0,1,2,3$.

Proof. Assume (i). Given $f, g \in D F(\mathfrak{X})$ and $u \in A$, there exist $F, F^{\prime}, G, G^{\prime} \in F^{\prime} R(\mathfrak{R})$ such that $i d_{A}=F^{\prime} \times F^{\prime}=G \times G^{\prime}$, and such that $f$ is the decomposition function associated with $F$ and $F^{\prime}$, and $g$ is the decomposition function associated with $G$ and $G^{\prime}$. By 4.5 we have

$$
(F|G| F) \cap F^{\prime} \subseteq G \quad \text { and } \quad\left(F \mid G^{\prime}\right) \cap F^{\prime} \subseteq G^{\prime} .
$$

For any $x \in A$,

$$
f_{u}(x) F x G g_{u}(x) F f_{u} g_{u}(x) \text { and } f_{u}(x) F^{\prime} u H^{\prime} f_{u}^{\circ} g_{u}(x),
$$

and therefore $f_{u}(x) G f_{u} g_{u}(x)$ or, equivalently, $g_{u} f_{u}(x)=g_{u} f_{u} g_{u}(x)$. Also,

$$
f_{u} g_{u}(x) F g_{u}(x) G^{\prime} u \text { and } f_{u} g_{u}(x) F^{\prime} u,
$$

so that $f_{u} g_{u}(x) G^{\prime} u$ or, equivalently, $g_{u} f_{u} g_{u}(x)=f_{u} g_{u}(x)$. Consequently, $f_{u} g_{u}(x)=g_{u} f_{u}(x)$, as was to be shown.

Clearly (ii) implies (iii). Finally assume (iii). To prove (i) it suffices according to 4.5 to prove that if

then

$$
i d_{A}=F \times F^{\prime}=G \times G^{\prime},
$$

$$
G \subseteq F^{\prime} \mid\left(F^{\prime} \cap G\right) \subseteq \underline{\left(F^{\prime} \cap G\right) \mid F} .
$$

Let $f$ and $g$ be the decomposition functions associated with $F$ and $F^{\prime}$ and with $G$ and $G^{\prime}$, respectively. Suppose $x G y$, and let $z=f(x, y)$. Choosing $u \in A$ so that

$$
f_{u} g_{u}(w)=g_{u} f_{u}(w) \quad \text { and } \quad f^{d}{ }_{u} g_{u}(w)=g_{u} f^{d} u(w) \quad \text { for } \quad w=x, y, z,
$$


observe that $g_{u}(x)=g_{u}(y), f_{u}(x)=f_{u}(z)$ and $f^{a}{ }_{u}(y)=f^{a}{ }_{u}(z)$. Hence

$$
\begin{aligned}
f_{u} g_{u}(y) & =f_{u} g_{u}(x)=g_{u} f_{u}(x)=g_{u} f_{u}(z)=f_{u} g_{u}(z), \\
f^{u}{ }_{u} g_{u}(y) & =g_{u} f^{d}{ }_{u}(y)=g_{u} f^{f}{ }_{u}(z)=f^{a}{ }_{u} g_{u}(z),
\end{aligned}
$$

whence it follows that $g_{u}(y)=g_{u}(z)$ or, equivalently, $z G y$. Thus $x F z\left(F^{\prime} \cap G^{\prime}\right) y$. We therefore see that the first inclusion in (1) holds.

Now suppose $x\left(F^{\prime}\left(F^{\prime} \cap G\right)\right) y$, and let $z=f(x, y)$ and $u=f(y, x)$. Since $z$ is the unique member of $A$ such that $x F z F^{\prime} y$, we must have $z G y$. Choosing $v \in A$. so that

$$
f_{v} g_{v}(w)=g_{v} f_{v}(w) \quad \text { and } \quad f^{d}{ }_{v} g_{v}(w)=g_{v} f_{v}^{d}(w) \quad \text { for } \quad w=x, y, z, u,
$$

observe that $f_{v}(z)=f_{v}(x), f_{v}^{d}(z)=f_{v}^{d}(y), f_{v}(u)=f_{v}(y), f_{v}^{d}(u)=f_{v}^{d}(x)$ and $g_{v}(z)=g_{v}(y)$. Consequently,

$$
\begin{gathered}
f_{v} g_{v}(u)=g_{v} f_{v}(u)=g_{v} f_{v}(y)=f_{v} g_{v}(y)=f_{v} g_{v}(z) \\
=g_{v} f_{v}(z)=g_{v} f_{v}(x)=f_{v} g_{v}(x), \\
f^{a} g_{v}(u)=g_{v} f_{v}^{a}(u)=g_{v} f_{v}^{a}(x)=f_{v}^{a} g_{v}(x),
\end{gathered}
$$

whence it follows that $g_{v}(u)=g_{v}(x)$ or, equivalently, $x G u$. We thus have $x\left(F^{\prime} \cap G\right) u F y$. This establishes the second inclusion in (1), and the proof of the theorem is complete.

6. The intermediate refinement property. We shall now introduce and study a property that is weaker than the strict refinement property but still implies, together with some mild additional conditions, that the given relational structure has the (ordinary) refinement property.

Definition 6.1. Given an element $u \in A$, we say that ( $\mathfrak{A}, u$ ) has the intermediate refinement property if and only if the following conditions are satisfied:

(i) For all $F, G \in F R(\mathfrak{U}), u /(F \mid G)=u /(G \mid F)$.

(ii) Fror all $F, F^{\prime}, G \in F R(\mathfrak{X})$, if $i d_{A}=F \times F^{\prime}$, then

$$
u /\left((F \mid G) \cap\left(F^{\prime} \mid G\right)\right)=u / G
$$

We say that $\mathfrak{A}$ has the intermediate refinement property if and only if (i) and (ii) hold for some $u \in A$.

It easily follows from 4.5 that $\mathfrak{U}$ has the strict refinement property if and only if $(\mathfrak{A}, u$ ) has the intermediate refinement property for every $u \in A$.
THEOREM 6.2. For any $u \in A$ the following conditions are equivalent:

(i) ( $\mathfrak{U}, u$ ) has the intermediate refinement property.

(ii) For all $F, F^{\prime}, G \in F R(\mathfrak{U})$, if $i d_{\Lambda}=F \times F^{\prime}$, then $u /(F \mid G)=u /(G \mid F)$ and $u /\left((F \mid G) \cap F^{\prime}\right) \subseteq u / G$.

(iii) For all $F, F^{\prime}, G \in F R(\mathfrak{A})$, if $i d_{A}=F \times F^{\prime}$, then $u /\left((F|G| F) \cap F^{\prime}\right)$ $\subset u / G$.

(iv) For all $F^{\prime}, F^{\prime}, G \in F R(\mathfrak{Y})$, if $i d_{d}=F \times F^{\prime}$, then $u \mid G \subseteq u /\left(F \mid\left(F^{\prime} \cap G\right)\right)$ $\subseteq u /\left(\left(F^{\prime} \cap G\right) \mid F\right)$.

Proof. The conditions (ii)-(iv) of the present theorem are obviously weaker forms of the conditions (iv)-(vi) of Theorem 4.5, and the proof given there of the equivalence of the latter three conditions can be imitated here to prove that (ii), (iii) and (iv) are equivalent.

If (i) holds, then under the hypothesis of (ii) we have

$$
u /\left((F \mid G) \frown F^{\prime}\right) \subseteq u /\left((F \mid G) \frown\left(F^{\prime} \mid G\right)\right)=u / G,
$$

and (ii) therefore is satisfied. Conversely, suppose that (ii) holds, and let $F, F^{\prime}$ and $G$ be as before. If $x \in u /\left((F \mid G) \cap\left(F^{\prime} \mid G\right)\right)$, then there exist $y, z \in A$ such that $u F y G x$ and $u F^{\prime} z G x$. Hence $u F y G z$ and $u F^{\prime} z$, whence it follows by (ii) that $u G z$. Since $z G x$, this implies that $u G x$. Thus $u /\left((F \mid G) \cap\left(F^{\prime} \mid G\right)\right)=u / G$, and (i) holds.

LEMMA 6.3. Suppose $u \in A$, and suppose that ( $\mathfrak{U}, u$ ) has the intermediate refinement property. If $G \in F R(\mathfrak{A})$ and $F_{i} \in F R(\mathfrak{U})$ for all $i \in I$, and if

then

$$
i d_{A}=\prod_{i \in I} F_{i}
$$

$$
u / G=\bigcap\left\{u /\left(F_{i} \mid G\right) \mid i \in I\right\} .
$$

Proof. There exist $G^{\prime} \in F R(\mathfrak{A})$ such that $i d_{A}=G \times G^{\prime}$, and consequently for each $i \in I$,

$$
u / F_{i}=u /\left(F_{i} \mid G\right) \cap u /\left(F_{i} \mid G^{\prime}\right) .
$$

If $x \in u /\left(F_{i} \mid G\right)$ for all $i \in I$, then we can find elements $y_{i} \in A$ such that $u G y_{i} F_{i} x$. Furthermore, there exists $z \in A$ such that $u G^{\prime} z G x$. For each $i \in I$ we have $u G y_{i} F_{i} x G z$ and $u G^{\prime} z$, whence if follows by 6.2 that $u F_{i} z$. Since this holds for all $i \in I$, we infer that $z=u$. Thus $u G x$ or, in other words, $x \in u / G$.

Lemma 6.4. If $u \in A$ is idempotent, and if $F, F^{\prime} \in F R(\mathfrak{U})$ are such that $i d_{A}=F \times F^{\prime}$, then $\mathfrak{A}(u / F) \cong \mathfrak{A} / F^{\prime}$.

Proof. For each $x \in u / F$ let $f(x)=x / F^{\prime \prime}$. For each $y \in A$ there exists $x \in A$ such that $u F x F^{\prime} y$, and hence $x \in u / F$ and $f(x)=y / F^{\prime}$. Thus $f$ maps $u / F$ onto $A / F^{\prime}$. If $x, y \in u / F$ and $f(x)=f(y)$, then $x F y$ and $x F^{\prime} y$, and 
hence $x=y$. Therefore $f$ is one-to-one. Finally, assuming that $t \in T$ and $x \epsilon^{e^{(t)}}(u / F)$, we have $x / F=z / F$ where $z=\langle u, u, \ldots, u\rangle$, and since $z \in R_{t}$ by hypothesis, this implies that $x / F \in R_{t} / F$. It therefore follows by 3.7 that the conditions $x \in R_{t}$ and $x / F^{\prime} \in R_{t} / F^{\prime}$ are equivalent. This shows that $f$ is an isomorphism.

Lemma 6.5. Suppose $u \in A, G \in F R(\mathfrak{U}), F_{i} \in F R(\mathfrak{A})$ for each $i \in I$, and

$$
i d_{A}=\prod_{i \in T} F_{i} .
$$

If $u / G=\bigcap\left\{u /\left(G \mid F_{i}\right) \mid i \epsilon I\right\}$, and if for each $i \in I$ we let $H_{i}$ be the restriction of $F_{i}$ to the set $B=u / G$, then in the substructure $\mathfrak{B}=\left\langle B, S_{t}\right\rangle_{t \in T}=\mathfrak{A}(B)$ of $\mathfrak{A}$ we have

$$
i d_{B}=\prod_{i \in I} H_{i} .
$$

Proof. It is clear that each $H_{i}$ is an equivalence relation over $B$, and that their intersection is the identity relation over $B$. For each $x \in{ }^{I^{I}} B$ there exists $v \in A$ such that $x_{i} F_{i} v$ for all $i \in I$. Thus $v$ belongs to all the sets $u /\left(G \mid F_{i}\right)$ and hence, by hypothesis, $v \in B$ and $x_{i} H_{i} v$ for all $i \in I$. Now suppose $t \in T$ and $x \epsilon^{e(t)} B$, and assume that $x / H_{i} \in S_{t} / H_{i}$ for all $i \in I$. Then it is easy to verify that $x / F_{i} \in R_{t} / F_{i}$ for all $i \in I$, whence it follows that $x \in R_{t}$ and, consequently, $x \in S_{t}$. The conclusion now follows by Theorem 3.4.

THEOREM 6.6. If there exists an idempotent element $u \in A$ such that $(\mathfrak{A}, u)$ has the intermediate refinement property, then $\mathfrak{U}$ has the refinement property.

Proof. If

$$
\mathfrak{U} \cong \mathbf{P}_{i \in I} \mathfrak{B}_{i} \text { and } \quad \mathfrak{U} \cong \mathbf{P}_{j \in J} \mathfrak{C}_{j},
$$

then by 3.1 and 3.2 there exist $F_{i}, G_{j} \in F R(\mathfrak{U})$, associated with all the elements $i \in I$ and $j \in J$, such that

$$
i d_{A}=\prod_{i \in T} F_{i}, \quad i d_{A}=\prod_{j \in J} G_{j},
$$

and such that $\mathfrak{B}_{i} \cong \mathfrak{U} / \mathbb{F}_{i}$ for all $i \in I$ and $\mathfrak{C}_{j} \cong \mathfrak{U} / G_{j}$ for all $j \in J$. For each $p \in I$ and $q \in J$, let

$$
\bar{F}_{p}=\prod_{p \neq i \in I} F_{i} \quad \text { and } \quad \bar{G}_{q}=\prod_{q \neq j \in J} G_{j},
$$

and let $G_{p, q}$ and $F_{p, q}$ be the restriction of $G_{q}$ to $u / \bar{F}_{p}$ and the restriction of $F_{p}$ to $u / \bar{G}_{q}$, respectively. Then, by 6.4 ,

$$
\mathfrak{B}_{p} \cong \mathfrak{A}\left(u / \bar{F}_{p}\right) \quad \text { and } \quad \mathfrak{G}_{q} \cong \mathfrak{U}\left(u / \bar{G}_{q}\right)
$$

and therefore, by 6.5 and 3.2 ,

$$
\mathfrak{B}_{p} \cong \mathbf{P}_{j \in J} \mathfrak{U}\left(u / \bar{F}_{p}\right) / G_{p, j} \quad \text { and } \quad \mathfrak{\complement}_{q} \cong \mathbf{P}_{i \in I} \mathfrak{A}\left(u / \bar{G}_{q}\right) / F_{i, q}
$$

Furthermore, letting

$$
\bar{F}_{p, q}=\prod_{p \neq i \in I} F_{i, q} \quad \text { and } \quad \bar{G}_{p, q}=\prod_{q \neq j \in J} G_{p, j}
$$

we again use 6.4 to infer that

$$
\mathfrak{U}\left(u / \bar{F}_{p}\right) / G_{p, q} \cong \mathfrak{A}\left(u / \bar{G}_{p, q}\right) \quad \text { and } \quad \mathfrak{A}\left(u / \bar{G}_{q}^{*}\right) / F_{p, q} \cong \mathfrak{U}\left(u / \bar{F}_{p, q}\right)
$$

Consequently

$$
\mathfrak{B}_{p} \cong \mathbf{P}_{j \in J} \mathfrak{A}\left(u / \bar{G}_{p, j}\right) \quad \text { and } \quad \widetilde{C}_{q} \cong \mathbf{P}_{i \in I} \mathfrak{A}\left(u / \bar{F}_{i, q}\right)
$$

The proof will therefore be complete if we show that, for all $p \in I$ and $q \in J, u / \bar{G}_{p, q}=u / \bar{F}_{p, q}$. But, in fact, the condition $x \in u / \bar{G}_{p, q}$ is equivalent to each of the following statements:

$$
\begin{array}{lll}
x \in u / G_{p, j} \quad \text { whenever } & q \neq j \in J, \\
x \in u / \bar{F}_{p} \text { and } x \in u / G_{j} & \text { whenever } & q \neq j \in J, \\
x \in u / F_{i} \text { and } x \in u / G_{j} & \text { whenever } & p \neq i \in I \text { and } q \neq j \in J .
\end{array}
$$

By symmetry, the condition $x \in u / \bar{F}_{p, q}$ is also equivalent to this last statement, and we therefore conclude that $u / \bar{G}_{p, q}=u / \bar{F}_{p, q}$.

THeonem 6.7. Suppose $u \in A$. Then ( $\mathfrak{A}, u$ ) has the intermediate refinement property if and only if, for all $f, g \in D F(\mathfrak{U})$ and $x \in A, f_{u} g_{u}(x)=u$ implies that $g_{u} f_{u}(x)=u$.

Proof. Assuming that this implication holds, we shall show that the condition $6.2(\mathrm{iv})$ is satisfied. This is equivalent to the assertions that, for all $F^{\prime}, F^{\prime}, G, G^{\prime} \in F^{\prime} R(\mathfrak{U})$ with $i d_{A}=F \times F^{\prime}=G \times G^{\prime}$, and for all $x \in A$, $u G x$ implies that $u F^{\prime} \mid\left(F^{\prime} \cap G\right) x$, $u F \mid\left(F^{\prime} \cap G\right) x$ implies that $u\left(F^{\prime} \cap G\right) \mid F x$.
Let $f$ and $g$ be the decomposition functions associated with the relations $F$ and $F^{\prime}$ and with $G$ and $G^{\prime}$, respectively. Assuming that $u G x$, let $z=f(u, x)$. Then $u F z F^{\prime} x$. Furthermore $g_{u}(x)=u$, hence $f^{d}{ }_{u} g_{u}(x)=u$, and therefore $g_{u} f^{d}{ }_{u}(x)=u$ or, in other words, $g_{u}(z)=u$. Thus $g_{u}(z)=g_{u}(x)$, whence it follows that $z G x$. Consequently $u F z\left(F^{\prime} \cap G\right) x$, and (1) is seen to hold.

Assuming now that $u F \mid\left(F^{\prime} \cap G\right) x$, let $z=f(u, x)$ and $v=f(x, u)$. Then $u F z F^{\prime} x$ and $z G x$. Since $g_{u} f_{u}(z)=g_{u} f_{u} f^{d} u(x)=u$, we have $f_{u} g_{u}(z)=u$. But $g_{u}(z)=g_{u}(x)$, so that this implies that $f_{u} g_{u}(x)=u$ and, consequently, $g_{u}(v)=g_{u} f_{u}(x)=u$. Thus $u G v$, and we conclude that $u\left(F^{\prime} \cap G\right) v F x$. This proves (2). 
Conversely, suppose that $(\mathfrak{H}, u)$ has the intermediate refinement property. Let the decomposition functions $f$ and $g$ be associated with the factor relations $F$ and $F^{\prime}$, and $G$ and $G^{\prime}$, respectively. For $x \in A$ the following conditions are equivalent:

$$
f_{u} g_{u}(x)=u, \quad g_{u}(x) F u, \quad u\left(F \cap G^{\prime}\right) g_{u}(x) G x, \quad x \in u /\left(\left(F \cap G^{\prime}\right) \mid G\right) .
$$

Similarly $g_{u} f_{u}(x)=u$ if and only if $x \in u /\left(\left(G \cap F^{\prime}\right) \mid F^{\prime}\right)$. To prove that $f_{u} g_{u}(x)=u$ implies $g_{u} f_{u}(x)=u$ it therefore suffices to show that $u /\left(\left(F \cap G^{\prime}\right) \mid G\right) \subseteq u /\left(\left(G \cap F^{\prime}\right) \mid F\right)$.

By 6.2 (iii) we have

$$
\left.u /\left(\left(G \cap F^{\prime}\right) \mid F^{\prime}\right) \supseteq u /\left(\left(F^{\prime}|G| F\right) \cap F^{\prime}\right) \mid F^{\prime}\right),
$$

and it follows by the modular law that

$$
\begin{aligned}
u /\left(\left(G \cap F^{\prime}\right) \mid F^{\prime}\right) & \supseteq u /\left(\left(F^{\prime}|G| F^{\prime}\right) \cap\left(F^{\prime} \mid F^{\prime}\right)\right)=u /(F|G| F) \\
& \supseteq u /\left(F^{\prime} \mid G\right) \supseteq u /\left(\left(F^{\prime} \cap G^{\prime}\right) \mid G\right) .
\end{aligned}
$$

7. Structures with a binary relation. The sufficient conditions that have been obtained so far for the existence of common refinements are not in such a form as to be easy to verify for specific structures. For such applications the criteria should be expressed in terms of the relations $R_{l}$ or in terms of some other relations $S$ obtained from these by means of some definite constructions. In general, if a new structure $\mathfrak{A}^{\prime}$ is constructed by adjoining to $\mathfrak{A}$ an $n$-ary relation $S$, then $F R\left(\mathfrak{Y}^{\prime}\right)$ $\supseteq F R(\mathfrak{U})$ and $D F\left(\mathfrak{U}^{\prime}\right) \supseteq D F(\mathfrak{U})$. In order for equalities to hold in these two formulas it is necessary and sufficient that the following condition be satisfied: For all $f \in D F(\mathfrak{I})$ and $x, y, z \epsilon^{n} A$, if $x, y \in S$ and if $z_{k}=f\left(x_{k}, y_{k}\right)$ for $k=0,1, \ldots, n-1$, then $z \in S$. If $S$ is defined in terms of the relations $R_{t}$ by means of a first order formula $\alpha$, conditions can be formulated in terms of the logical structure of $\alpha$ that will insure that $S$ has this property. Actually we shall describe the admissible constructions in purely mathematical terms, and for this purpose some additional notation is needed.

By the juxtaposition of an $m$-termed sequence $x$ and an $n$-termed sequence $y$-in symbols $x-y$ - we mean the $(m+n)$-termed sequence $\left\langle x_{0}, x_{1}, \ldots, x_{m-1}, y_{0}, y_{1}, \ldots, y_{n-1}\right\rangle$. If $s_{1}$ and $s_{2}$ are two relations, then we let $S_{1}-S_{2}$ be the relation consisting of all sequences of the form $x-y$ with $x \in S_{1}$ and $y \in S_{2}$. If $n>1$, and if $S$ is an $n$-ary relation whose field is contained in $A$, then we let $P(S)$ and $Q(S)$ be the $(n-1)$-ary relations such that, for all $x \epsilon^{(n-1)} A$,

$x \in P(S)$ if and only if $x^{-}\langle y\rangle \in S$ for some $y \in A$,

$x \in Q(S)$ if and only if $x^{-}\langle y\rangle \in S$ for all $y \in A$
If $i, j$ and $n$ are natural numbers with $i<j<n$, then we let $I_{i, j, n}$, be the set of all $x \in \epsilon^{n} A$ such that $x_{i}=x_{j}$. Finally, if $s^{\prime}$ in an $n$-ary relation, and if $\varphi$ is a permutation of the set $n=\{0,1, \ldots, n-1\}$, then we let $S \varphi$ be the $n$-ary relation consisting of all sequences of the form $x \circ \varphi=\left\langle x_{\varphi(0)}, x_{\varphi(1)}, \ldots, x_{\varphi(n-1)}\right\rangle$ with $x \in S$.

DeFinition 7.1. By $\Delta(\mathfrak{A})$ we mean the intersection of all families $\mathscr{F}$ of relations whose field is contained in $A$ having the following properties:

(i) $R_{l} \in \mathcal{F}$ for all $t \in T,{ }^{n} A \in \mathcal{T}$ for all positive integers $n, I_{i, j, n} \in \mathcal{F}$ whenever $i, j, n$ are natural numbers with $i<j<n$.

(ii) For any $n$-ary relation $S$ with $n>1$, if $S \in \mathcal{F}$ then $P(S) \in \mathbb{F}$ and $Q(S) \in \mathcal{F}$ and $S \varphi \in \mathcal{F}$ whenever $\phi$ is a permutation of $n$.

(iii) For any $S_{1}, S_{2} \in \mathcal{F}, S_{1}-S_{2} \in \mathcal{F}$ and if $S_{1}$ and $S_{2}$ are relations of the same rank, then $S_{1} \cap S_{2} \in \mathbb{F}$.

Corollary 7.2. Suppose $S \subseteq^{n} A$ and $S \in \Delta(\mathfrak{U})$. For all $f \in D F(\mathfrak{U})$ and $x, y, z, \epsilon^{n} A$, if $x, y \in S$ and if $z_{k}=f\left(x_{k}, y_{k}\right)$ for $k=0,1, \ldots, n-1$, then $z \in S$.

Proof. It is a simple matter to check that the class $\widetilde{F}$ of all relations $S$ with the above property satisfies the conditions 7.1 (i)-(iii), whence it follows that $\Delta(\mathfrak{U}) \subseteq \mathscr{T}$.

For the formulation and proof of the next theorem, which is the principal result in this section, some additional terminology is needed. Suppose that $S$ is a binary relation. An element $u$ in the field of $S$ is said to be antisymmetric with respect to $S$ if, for every element $x$, the conditions $u S x$ and $x S u$ jointly imply that $x=u$. A finite sequence $z=\left\langle z_{0}, z_{1}, \ldots, z_{n}\right\rangle$ is said to $S$-connect two elements $x$ and $y$ if $x=z_{0}$ and $y=z_{n}$ and, for each $i<n$, either $z_{i} S z_{i+1}$ or $z_{i+1} S z_{i}$. We say that $x$ and $y$ are $(S, n)$-comnected if there exists an $(n+1)$-termed sequence that $S$-connects $x$ and $y$, and we say that $x$ and $y$ are $S$-connected if they are $(S, n)$-connected for some natural number $n$. Finally, we say that the relation $S$ is connected if any two members of the field of $S$ are $S$-connected.

THeOREm 7.3. Suppose $u \in A$. If there exists a reflexive and comnected binary relation $S \in A(\mathfrak{O})$ such that the field of $S$ is $A$ and $u$ is antisymmetric with respect to $S$, then (2F, $u$ ) has the intermediate refinement property.

Proof. Although $S$ is not assumed to be a partially ordering relation, it is suggestive to write $x \leqslant y$ or, equivalently, $y \geqslant x$ for $x S y$. Let $\equiv$ be the smallest equivalence relation over $A$ such that the conditions $x \leqslant y$ and $y \leqslant x$ jointly imply that $x \equiv y$, and observe that $x \equiv u$ implies that $x=u$. For $v \in A$ let $C(v)$ be the set of all elements $x \in A$ with the property that $f_{v} g_{v}(x) \equiv g_{v} f_{v}(x)$ for all $f, g \in D F(\mathfrak{A})$. 
We shall prove by induction on the pcsitive integer $n$ that the following statement holds for all $x, v \in A$ :

(1) If $x$ and $v$ are $(S, n)$-connected, then $x \in C(v)$.

Once this has been established the theorem readily follows. In fact, since it is assumed that $S$ is connected and that the field of $S$ is $A$, (1) implies that $C(v)=A$ for all $v \in A$, and hence in particular $O(u)=A$. Consequently, if $f, g \in D F(\mathfrak{A})$, and if $x \in A$ is such that $f_{u} g_{u}(x)=u$, then $g_{u} f_{u}(x) \equiv u$, and therefore $g_{u} f_{u}(x)=u$. From this the conclusion follows by 6.7 .

Observe that, for all $f \in D F(\mathfrak{U})$ and $x, y, z, x^{\prime}, y^{\prime}, v \in A$, the following statements hold:

(2) If $x \leqslant x^{\prime}$ and $y \leqslant y^{\prime}$, then $f(x, y) \leqslant f\left(x^{\prime}, y^{\prime}\right)$.

(3) If $x \leqslant y$, then $f_{v}(x) \leqslant f_{v}(y)$.

(4) If $x \leqslant z$ and $y \leqslant z$, then $f(x, y) \leqslant z$.

(5) If $x \equiv x^{\prime}$ and $y \equiv y^{\prime}$, then $f(x, y) \equiv f\left(x^{\prime}, y^{\prime}\right)$.

(6) If $f_{v}(x) \leqslant f_{v}(y)$ and $f^{d}{ }_{v}(x) \leqslant f^{d}{ }_{v}(y)$, then $x \leqslant y$.

(7) If $f_{v}(x) \equiv f_{v}(y)$ and $f^{d}{ }_{v}(x) \equiv f^{d}{ }_{v}(y)$, then $x \equiv y$.

In fact, (2) is simply a translation of 7.2 into the present notation, (3) follows from (2) and the fact that $v \leqslant v$, and (4) is obtained from (2) by recalling that $f(z, z)=z$. Of course the dual of (4), obtained by writing $\geqslant$ for $\leqslant$, also holds. If $x \equiv x^{\prime}$ and $y \equiv y^{\prime}$, then there exist $r_{0}, r_{1}, \ldots, r_{m}$, $s_{0}, s_{1}, \ldots, s_{n} \in A$ such that $x=r_{0}, x^{\prime}=r_{m}, y=s_{0}$ and $y^{\prime}=s_{n}$, and such that $r_{i} \leqslant r_{i+1}$ and $r_{i+1} \leqslant r_{i}$ for $i=0,1, \ldots, m-1$, and $s_{j} \leqslant s_{j+1}$ and $s_{j+1} \leqslant s_{j}$ for $j=0,1, \ldots, n-1$. Since $y \leqslant y$, we infer by $(2)$ that $f\left(r_{i}, y\right)$ $\leqslant f\left(r_{i+1}, y\right)$ and $f\left(r_{i+1}, y\right) \leqslant f\left(r_{i}, y\right)$ for $i=0,1, \ldots, n-1$, and from this it follows that $f(x, y) \equiv f\left(x^{\prime}, y\right)$. Similarly $f\left(x^{\prime}, y\right) \equiv f\left(x^{\prime}, y^{\prime}\right)$, and we therefore have $f(x, y) \equiv f\left(x^{\prime}, y^{\prime}\right)$. This proves (5). Finally, (6) and (7) are obtained from (2) and (5), respectively, by using the formula $f\left(f_{v}(x), f^{d} v(x)\right)=x$, and the corresponding formula with $x$ replaced by $y$. We next prove the following statement:

(8) If $x, v \in C(w)$ and if $f_{v}(x), g_{v} f_{v}(x) \in C(w)$ for all $f, g \in D F(\mathfrak{U})$, then $x \in O(v)$.

By several applications of the hypothesis and of 5.5 (ii), (iii) we find that

$$
\begin{aligned}
f_{w} g_{w} f_{v} g_{v}(x) & \equiv g_{w} f_{w} f_{v} g_{v}(x)=g_{w} f_{w} g_{v}(x) \equiv f_{w} g_{w} g_{v}(x)=f_{w} g_{w}(x), \\
f_{w} g_{w} g_{v} f_{v}(x) & =f_{w} g_{w} f_{v}(x) \equiv g_{w} f_{w} f_{v}(x)=g_{w} f_{w}(x) \equiv f_{w} g_{w}(x), \\
f^{d}{ }_{w} g_{w} f_{v} g_{v}(x) & \equiv g_{w} f^{d}{ }_{w} f_{v} g_{v}(x)=g_{w} f^{d}{ }_{w}(v), \\
f^{d}{ }_{w} g_{w} g_{v} f_{v}(x) & =f^{d}{ }_{w} g_{w} f_{v}(x) \equiv g_{w} f^{d}{ }_{w} f_{v}(x)=g_{w} f^{d}{ }_{w}(v) .
\end{aligned}
$$

\section{Consequently}

$$
f_{w} g_{w} f_{v} g_{v}(x) \equiv f_{w} g_{w} g_{v} f_{v}(x) \quad \text { and } \quad f^{d}{ }_{w} g_{w} f_{v} g_{v}(x) \equiv f^{d}{ }_{w} g_{w} g_{v} f_{v}(x),
$$

whence it follows by (7) that

$$
g_{w} f_{v} g_{v}(x) \equiv g_{w} g_{v} f_{v}(x) \text {. }
$$

A similar argument shows that

$$
g^{d}{ }_{w} f_{v} g_{v}(x) \equiv g^{d}{ }_{w} g_{v} f_{v}(x)
$$

and we infer by a second application of (7) that $f_{v} g_{v}(x) \equiv g_{v} f_{v}(x)$. Since $f$ and $g$ are arbitrary members of $D F(\mathfrak{H})$, we conclude that $x \in C(v)$.

We are now ready to begin the proof of (1). First assume that $x$ and $v$ are $(S, 1)$-connected; by symmetry we may assume $v \leqslant x$. Applying (2) and (3) several times and (4) once, we obtain $v=f(v, v)$ $\leqslant f(x, v) \leqslant f(x, x), v \leqslant f_{v}(x) \leqslant x$, and thence $v=g_{v}(v) \leqslant g_{v} f_{v}(x) \leqslant g_{v}(x)$ and also $g_{v} f_{v}(x) \leqslant f_{v}(x)$. Using the dual of (4) we infer that

$$
g_{v} f_{v}(x) \leqslant f\left(g_{v}(x), f_{v}(x)\right)=f_{v} g_{v}(x) .
$$

Similarly $f_{v} g_{v}(x) \leqslant g_{v} f_{v}(x)$, and therefore $f_{v} g_{v}(x) \equiv g_{v} f_{v}(x)$. The statement (1) therefore holds for $n=1$.

Next suppose $n>1$, and assume that (1) holds whenever $n$ is replaced by a smaller integer. Suppose the sequence $z=\left\langle z_{0}, z_{1}, \ldots, z_{n}\right\rangle S$-connects $x$ and $v$. Letting $w=z_{n-1}$ we may assume that $v \leqslant v$. By the inductive hypothesis and by the case already proved we have $x, v \in C(w)$. It follows from (8) that in order to prove (1) for the present case it suffices to show that if $f$ and $g$ are any members of $D F(\mathfrak{U})$, then $f_{v}(x)$ and $g_{v} f_{v}(x)$ also belong to $C(w)$. We consider two cases.

Case 1. For some $j \leqslant n-1, z_{j} \geqslant z_{j+1}$. So define the sequence $z^{\prime}=\left\langle z_{0}^{\prime}, z_{1}^{\prime}, \ldots, z_{n-1}^{\prime}\right\rangle$ that $z_{i}^{\prime}=f_{v}\left(z_{i}\right)$ for $i \leqslant j$ and $z_{i}^{\prime}=f_{w}\left(z_{i}\right)$ whenever $j<i<n$. From the fact that $z_{j} \geqslant z_{j+1}$ and $v \geqslant w$ we infer by (2) that $z_{j}^{\prime} \geqslant z_{j+1}^{\prime}$, and it is therefore easily seen that $z^{\prime} S$-connects $f_{v}(x)$ and $w$. Similarly, letting $z_{i}^{\prime \prime}=g_{v} f_{v}\left(z_{i}\right)$ for $i \leqslant j$ and $z_{i}^{\prime \prime}=g_{w} f_{w}\left(z_{i}\right)$ whenever $j<i<n$ we find that the sequence $z^{\prime \prime}=\left\langle z_{0}^{\prime \prime}, z_{1}^{\prime \prime}, \ldots, z_{n-1}^{\prime \prime}\right\rangle S$-connects $g_{v} f_{v}(x)$ and $w$. By the inductive hypothesis we therefore see that $f_{v}(x)$ and $g_{v} f_{v}(x)$ belong to $C(w)$ in this case.

Case 2. $z_{j} \leqslant z_{j+1}$ for all $j<n$. In this case

$$
\begin{gathered}
f_{v}(x)=f_{v}\left(z_{0}\right) \leqslant f_{v}\left(z_{1}\right) \leqslant \ldots \leqslant f_{v}\left(z_{n-1}\right)=f_{v}(w) \geqslant w, \\
g_{v} f_{v}(x)=g_{v} f_{v}\left(z_{0}\right) \leqslant g_{v} f_{v}\left(z_{1}\right) \leqslant \ldots \leqslant g_{v} f_{v}\left(z_{n-1}\right)=g_{v} f_{v}(w) \geqslant w,
\end{gathered}
$$

and we may apply the dual of Case 1 twice, replacing $v$ and $x$ by $w$ and $f(x)$ in one case and by $w$ and $g_{v} f_{v}(x)$ in the other, to infer that $f_{v}(x)$ and $g_{v} f_{v}(x)$ belong to $C(w)$.

The theorem now follows by induction. 
COROLLARY 7.4. Suppose that there exists an antisymmetric, reflexive and connected binary relation $S \in \Delta(\mathfrak{H})$ such that the field of $S$ is $A$. Then $\mathfrak{A}$ has the strict refinement property.

Proof. By 7.3 and the remark following 6.1 .

It is known that in this last result we cannot omit the assumption that $S$ be connected. In fact, there exist finite structures $\mathfrak{A}=\langle A, R\rangle$ with a binary relation $R$ that is reflexive and antisymmetric, and whose field is $A$, which do not have the unique factorization property. It is still an open question whether for such structures the condition $\mathfrak{A} \times \mathfrak{A}$ $\cong \mathfrak{B} \times \mathfrak{B}$ implies that $\mathfrak{A} \cong \mathfrak{B}$.

These structures do however have a unique decomposition property of a different kind that can be obtained with the aid of 7.4. Given a system of structures $\mathfrak{B}_{i}=\left\langle B_{i}, S_{i}\right\rangle, i \in I$, of the type under consideration, if the sets $B_{i}$ are pairwise disjoint, then we let

$$
\sum_{i \in I} \mathfrak{B}_{i}=\left\langle\bigcup_{i \in I} B_{i}, \bigcup_{i \in I} S_{i}\right\rangle
$$

and call this new structure the cardinal sum of the structures $\mathfrak{B}_{i}$. A structure $\mathfrak{A}=\langle A, R\rangle$ is said to be additively indecomposable if it is not a cardinal sum of proper substructures. It is obvious that $\mathfrak{A}$ is additively indecomposable if and only if $S$ is connected. It is also easy to show that $\mathfrak{U}$ is always the cardinal sum of additively indecomposable substructures, and that this representation is unique except for the order of the summands. From these observations together with 7.4 we obtain the following result:

Suppose $\mathfrak{A}=\langle A, R\rangle$ is a fin itestructure such that $R$ is an antisymmetric, reflexive binary relation whose field is $A$. Then $\mathfrak{A}$ has up to isomorphism a unique representation

$$
\mathfrak{A} \cong \sum_{i \in I} \underset{j \in J_{i}}{\mathbf{P}} \mathfrak{B}_{i, j}
$$

where each of the structures $\mathfrak{B}_{i, j}=\left\langle B_{i, j}, S_{i, j}\right.$ is directly indecomposable and all the relations $S_{i, j}$ are connected.

Returning now to arbitrary $\varrho$-structures $\mathfrak{A}$, if + is a binary operation that belongs to $\Delta(\mathfrak{A})$, then this operation can be used to define various binary relations $S$ in $\Delta(\mathfrak{U})$. If one of these relations satisfies the conditions of 7.4 , then we can of course infer that $\mathfrak{A}$ has the strict refinement property. This is illustrated in Corollary 7.5 below. It is also possible to derive in this manner a number of results, both old and new, concerning algebras with a zero element. However, these theorems are special cases of results obtained in the next section by more direct methods, and will therefore not be given here (cf. $8.3,8.5$ and 8.8 )
COROLLARY 7.5. Suppose that there exists a binary operation + in $\Delta(\mathfrak{H})$ such that $x+x=x$ for all $x \in A$, and such that for all $x, y \in A$ one of
the following conditions holds:

(i) There exists $z \in A$ such that $x+z=z+x=x$ and $y+z=z+y=y$.

(ii) There exists $z \in A$ such that $x+z=z+x=z$ and $y+z=z+y=z$.

Then $\mathfrak{A}$ has the strict refinement property.

Proof. Let $S$ be the binary relation such that, for all $x, y, x S y$ if and only if $x, y \in A$ and $x+y=y+x=x$. This relation is easily seen to satisfy the conditions in 7.4, whence the conclusion follows.

8. Structures with a binary operation. A great deal is known concerning refinement properties of algebraic structures with a binary operation + and a zero element 0 , such that $0+x=x=x+0$ for all $x \in A$. In particular, it is known that the strict refinement property holds whenever 0 is a strong zero element ([10], p. 277), and, more generally, this is known to be the case whenever $\mathfrak{A}$ is centerless ([8], p. 54), or zero equivalent ([5], p. 308). In this section we shall generalize some of these results in two directions. First, we do not assume that all the relations $R_{t}$ of the given system $\mathfrak{U}=\left\langle A, R_{t}\right\rangle_{t \in T}$ are operations, and instead of assuming that the operation + is one of these relations we only require it to be a member of $\Delta(\mathfrak{A})$. Secondly, and more significantly, we shall show that the condition $0+x=x=x+0$ can be replaced by various combinations of weaker assumptions. We begin by listing for convenient reference the various properties that will be considered in this connection, denoting the distinguished element by the letter $u$ rather than by the symbol 0 :

$\left(\mathrm{Z}_{1}\right) \quad u+u=u$.

$\left(Z_{2}\right)$ For all $x \in A, u+x=x+u$.

$\left(\mathrm{Z}_{3}\right)$ For all $x, y \in A, u+(x+y)=(u+x)+y, x+(u+y)=(x+u)+y$, and $x+(y+u)=(x+y)+u$.

$\left(\mathrm{Z}_{4}\right)$ For all $x, y \in A$, if $u+x=u+y$, then $x=y$.

$\left(Z_{5}\right)$ Fror all $x \in A$, if $u+x=u+u$, then $x=u$.

$\left(Z_{6}\right)$ For all $x, y \in A$, if $x+y=u+u$, then $x=y=u$.

LEMCMA 8.1. Suppose that the binary operation + in $\Delta(\mathfrak{A})$ and the element $u \in A$ satisfy $\left(Z_{2}\right)$. Then for all $f, g \in D F(\mathfrak{A})$ and $x, y \in A$ the following conditions hold:
(i) $f_{u}(x)+f^{a} u(x)=u+x$.
(ii) $f_{u} g_{u} f_{u}^{d}(x)+f_{u} g_{u}^{d} f^{d}{ }_{u}(x)=u+u$.
(iii) $f_{u} g_{u} f^{d}{ }_{u}(x)+g_{u} f_{u}(x)=f_{u}^{d} g_{u} f_{u}(x)+f_{u} g_{u}(x)$.
(iv) $f_{u}(x)+f^{a}{ }_{u}(y)=f^{a} u(y)+f_{u}(x)$.

Fundamenta Mathematicae, T. LV 
(v) $x+y=y+x$ if and only if $f_{u}(x)+y=y+f_{u}(x)$ and $f_{u}^{a}(x)+y$ $=y+f^{d}{ }_{u}(x)$.

(vi) $x+y=y+x$ if and only if $f_{u}(x)+f_{u}(y)=f_{u}(y)+f_{u}(x)$ and $f^{d}{ }_{u}(x)+f^{d}{ }_{u}(y)=f^{d}{ }_{u}(y)+f^{d} u(x)$.

(vii) $f_{u} g_{u} f_{u}^{a}(x)+y=y+f_{u} g_{u} f_{u}^{a}(x)$.

Proof. Applying 7.2 with + in place of the relation $S$, we see that for all $x, y, x^{\prime}, y^{\prime} \in A$

$$
f(x, y)+f\left(x^{\prime}, y^{\prime}\right)=f\left(x+x^{\prime}, y+y^{\prime}\right)
$$

and hence in particular

$$
f_{u}(x)+f_{u}(y)=f_{u+u}(x+y) .
$$

Using (1) together with 5.1 (i) we find that

$f_{u}(x)+f^{d} u(x)=f(x, u)+f(u, x)=f(x+u, u+x)=f(u+x, u+x)=u+x$,

proving (i). Using this together with (2), 5.1 (i) and 5.5 (iii) we compute

$$
\begin{gathered}
f_{u} g_{u} f_{u(x)}^{a}+f_{u} g_{u}^{d} f_{u}^{d}(x)=f_{u+u}\left(g_{u} f_{u}^{d}(x)+g_{u}^{d} f^{d}{ }_{u}(x)\right) \\
=f_{u+u}\left(u+f_{u}^{d}(x)\right)=f_{u}(u)+f_{u} f_{u}^{d}(x)=u+u .
\end{gathered}
$$

Thus (ii) holds. Using (1), (2) and (i) together with 5.1 (i) and $\left(Z_{2}\right)$ we further compute

$$
\begin{aligned}
& f_{u} g_{u} f^{d}{ }_{u}(x)+g_{u} f_{u}(x)=f\left(g_{u} f_{u}^{d}(x), u\right)+f\left(g_{u} f_{u}(x), g_{u} f_{u}(x)\right) \\
& =f\left(g_{u} f_{u}^{d}(x)+g_{u} f_{u}(x), u+g_{u} f_{u}(x)\right)=f\left(g_{u+u}\left(f^{d}{ }_{u}(x)+f_{u}(x)\right), u+g_{u} f_{u}(x)\right) \\
& =f\left(g_{u+u}(u+x), u+g_{u} f_{u}(x)\right)=f\left(g_{u}(u)+g_{u}(x), u+g_{u} f_{u}(x)\right) \\
& =f\left(u+g_{u}(x), g_{u} f_{u}(x)+u\right)=f\left(u, g_{u} f_{u}(x)\right)+f\left(g_{u}(x), u\right) \\
& =f_{u}^{d} g_{u} f_{u}(x)+f_{u} g_{u}(x) .
\end{aligned}
$$

This proves (iii). From (1) we infer that

$$
\begin{aligned}
& f_{u}(x)+f_{u}^{a}(y)=f(x+u, u+y), \\
& f_{u}^{d}(y)+f_{u}(x)=f(u+x, y+u),
\end{aligned}
$$

whence (iv) follows by $\left(\mathrm{Z}_{2}\right)$. If $x+y=y+x$, then by 5.1 (i) and (1),

$$
\begin{aligned}
f_{u}(x)+y & =f(x, u)+f(y, y)=f(x+y, u+y)=f(y+x, y+u) \\
& =f(y, y)+f(x, u)=y+f_{u}(x)
\end{aligned}
$$

and, similarly, $f^{d} u(x)+y=y+f^{d} u(x)$. Conversely, if $y$ commutes with $f_{u}(x)$ and $f_{u}^{d}(x)$, then we use the fact that, by 5.1 (i), (ii),

$$
x=f\left(f_{u}(x), f_{u}^{d}(x)\right), \quad y=f(y, y),
$$

together with (1) to infer that $x$ and $y$ ccmmute. Hence $(v)$ holds. Next, applying $(\nabla)$ twice we see that $x$ and $y$ commute with each other if and only if each of the elements $f_{u}(x), f^{d}{ }_{u}(x)$ ec mmutes with each of the elements $f_{u}(y), f_{u}^{d}(y)$. Since, by (iv), $f_{u}(x)$ c c mmutes with $f^{a} u(y)$ and $f^{d} u(x)$ commutes with $f_{u}(y)$, this yields (vi). Finally, by (iv), $f_{u}^{a}(x)$ commutes with $f_{u}(y)$, whence it folows by $(v)$ that $f_{u} g_{u} f_{u}^{d}(x)$ commutes with $f_{u}(y)$. Since, by (iv), $f_{u} g_{u} f^{d}{ }_{u}(x)$ also commutes with $f_{u}^{d}(y)$, we conclude with the aid of $(\nabla)$ that $f_{u} g_{u} f^{d}{ }_{u}(x)$ commutes with $y$.

LEMMA 8.2. Suppose that the binary operation + in $\Delta(\mathfrak{H})$ and the element $u \in A$ satisfy $\left(\mathrm{Z}_{2}\right)$ and $\left(\mathrm{Z}_{5}\right)$. Then ( $\left.\mathfrak{A}, u\right)$ has the intermediate refinement property if and only if $f_{u} g_{u} f^{d}{ }_{u}(x)=u$ for all $f, g \in D F(\mathfrak{A})$ and $x \in A$.

Proof. By $6.7,(\mathfrak{A}, u)$ has the intermediate refinement property if and only if, for all $f, g \in D F^{\prime}(\mathfrak{Q})$ and $x \in A$,

$$
g_{u} f_{u}(x)=u \quad \text { implies } \quad f_{u} g_{u}(x)=u \text {. }
$$

Since $g_{u} f_{u} f_{u}^{d}(x)=u$, (1) implies that $f_{u} g_{u} f_{u}^{d}(x)=u$. Conversely, suppose that this last formula holds, and assume that $g_{u} f_{u}(x)=u$. Together with 8.1 (iii) this yields $u+u=u+f_{u} g_{u}(x)$, whence it follows by $\left(Z_{5}\right)$ that $f_{u} g_{u}(x)=u$. Thus (1) is satisfied.

THEOREM 8.3. Suppose that there exist a binary operation + in $\Delta(\mathfrak{U})$ and an idempotent element $u \in A$ such that $\left(Z_{2}\right)$ and $\left(Z_{6}\right)$ hold. Then $\mathfrak{A}$ has the refinement property.

Proof. For all $f, g \in D F(\mathfrak{U})$ and $x \in A$ we have

$$
f_{u} g_{u} f_{u}^{d}(x)+f_{u} g_{u}^{d} f^{d} u(x)=u+u
$$

by 8.1 (ii), and hence $f_{u} g_{u} f^{d}(x)=u$ by $\left(\mathrm{Z}_{6}\right)$. Consequently $(\mathfrak{A}, u)$ has the intermediate refinement property, and the conclusion follows by 6.6 .

THEOREM 8.4. Suppose that the binary operation + in $\Delta(\mathfrak{2})$ and the element $u \in A$ satisfy $\left(\mathrm{Z}_{2}\right)$ and $\left(\mathrm{Z}_{4}\right)$. Then $\mathfrak{A}$ has the strict refinement property $i_{f}^{f}$ and only if ( $\mathfrak{A}, u$ ) has the intermediate refinement property.

Proof. If $(\mathfrak{A}, u)$ has the intermediate refinement property, then for all $f, g \in D F^{\prime}(\mathfrak{U})$ and $x \in A$ we have $f_{u} g_{u} f^{d}{ }_{u}(x)=u$ by 8.2 and hence $f_{u} g_{u}(x)=g_{u} f_{u}(x)$ by 8.1 (iii) and $\left(\mathrm{Z}_{4}\right)$. According to 5.6 this implies that $\mathfrak{A}$ has the strict refinement property. The implication in the other direction holds for every relational system $\mathfrak{A}$. 
COROLLARY 8.5. Suppose that there exist a binary operation + in $\Delta(\mathfrak{I})$ and an element $u \in A$ such that $\left(\mathrm{Z}_{2}\right),\left(\mathrm{Z}_{4}\right)$ and $\left(\mathrm{Z}_{6}\right)$ hold. Then $\mathfrak{A}$ has the strict refinement property.

Proof. We see as in the proof of 8.3 that $\left(Z_{2}\right)$ and $\left(Z_{6}\right)$ imply that $(\mathfrak{A}, u)$ has the intermediate refinement property. Hence the conclusion follows by 8.4 .

This corollary is a generalization of the theorem that asserts that every algebra with a strong zero element has the strict refinement property. In order to obtain similar generalizations of the ccrresponding theorems for centerless algebras and for zero-equivalent algebras we must investigate furthermore the maps $f_{u} g_{u} f^{d}{ }_{u}$. We shall only consider here a counterpart of the first of these theorems.

It has already been shown, in 8.1 (vi), that if $u$ commutes with every element of $A$, then each element of the form $z^{\prime}=f_{u} g_{u} f^{d}{ }_{u}(z)$ also commutes with all the members of $A$. It will now be shown that similar statements hold concerning the conditions $\left(Z_{3}\right)$ and $\left(Z_{4}\right)$, in the latter case under the assumption that $\left(Z_{2}\right)$ and $\left(Z_{3}\right)$ are also satisfied.

LEMNA 8.6. Suppose that the binary operation + in $\Delta(\mathfrak{A})$ and the element $u \in A$ satisfy $\left(\mathrm{Z}_{3}\right)$. If $f, g \in D F^{\prime}(\mathfrak{U})$ and $z \in A$, then the conditions obtained from $\left(\mathrm{Z}_{3}\right)$ by replacing $u$ by $f_{u} g_{u} f^{d}{ }_{u}(z)$ are satisfied.

Proof. We shall verify only the condition obtained from the last formula in $\left(Z_{3}\right)$. This condition asserts that the three elements $x, y, f_{u} g_{u} f^{d}{ }_{u}(z)$ associate; i.e., that

$$
x+\left(y+f_{u} g_{u} f^{d}{ }_{u}(z)\right)=(x+y)+f_{u} g_{u} f^{d}{ }_{u}(z) .
$$

The proof will be based on three statements which hold for all $f \in D F(\mathfrak{A})$ and $x, y, z \in A$ :

$$
\text { I. } x, y, z \text { associate if and only if } f_{u}(x), f_{u}(y), f_{u}(z) \text { associate and }
$$
$f^{d}{ }_{u}(x), f^{d}{ }_{u}(y), f^{d}{ }_{u}(z)$ associate.

II. $f_{u}(x), f_{u}(y)$ and $f_{u}^{d}(z)$ associate.

III. If $x, y$ and $z$ associate, then $x, y$ and $f_{u}(z)$ associate.

First observe that (1) readily follows from these three statements. In fact, since $f_{u} f_{u} g_{u} f^{d}{ }_{u}(z)=f_{u} g_{u} f^{l}{ }_{u}(z)$ and $f_{u}^{d} f_{u} g_{u} f^{d}{ }_{u}(z)=u$, and since $f_{u}^{d}(x), f_{u}^{d}(y)$ and $u$ associate, we see by $I$ that (1) reduces to showing that $f_{u}(x), f_{u}(y), f_{u} g_{u} f^{d} u(z)$ associate. But $f_{u}(x), f_{u}(y), f^{d}{ }_{u}(z)$ associate by II, and applying III twice we obtain the desired conclusion.

Let $v=u+(u+u)=(u+u)+u$, and observe that

$$
\begin{aligned}
& f_{v}(x+(y+z))=f_{u}(x)+\left(f_{u}(y)+f_{u}(z)\right), \\
& f_{v}((x+y)+z)=\left(f_{u}(x)+f_{u}(y)\right)+f_{u}(z) .
\end{aligned}
$$

From these formulas and the corresponding formulas with $f_{v}$ replaced by $f_{v}^{d}$ the statement I follows by 5.5 (vii).

By easy calculations we obtain

$$
\begin{aligned}
f_{v}\left(f_{u}(x)+\left(f_{u}(y)+f^{d}{ }_{u}(z)\right)\right) & =f_{u}(x)+\left(f_{u}(y)+u\right), \\
f_{v}\left(\left(f_{u}(x)+f_{u}(y)\right)+f_{u}^{d}(z)\right) & =\left(f_{u}(x)+f_{u}(y)\right)+u, \\
f^{d}{ }_{v}\left(f_{u}(x)+\left(f_{u}(y)+f^{a}{ }_{u}(z)\right)\right) & =u+\left(u+f_{u}^{d}(z)\right), \\
f^{u}{ }_{v}\left(\left(f_{u}(x)+f_{u}(y)\right)+f_{u}^{d}(z)\right) & =(u+u)+f_{u}^{d}(z),
\end{aligned}
$$

and since the associative law holds whenever one of the three elements involved is $u$, we again apply 5.5 (vii) and conclude that II holds.

Finally we have

$$
\begin{gathered}
f_{v}\left(x+\left(y+f_{u}(z)\right)\right)=f_{u}(x)+\left(f_{u}(y)+f_{u}(z)\right), \\
f_{v}\left((x+y)+f_{u}(z)\right)=\left(f_{u}(u)+f_{u}(y)\right)+f_{u}(z), \\
f_{v}^{d}\left(x+\left(y+f_{u}(z)\right)\right)=f_{u}^{d}(x)+\left(f^{d} u(y)+u\right), \\
f_{v}^{d}\left((x+y)+f_{u}(z)\right)=\left(f_{u}^{d}(x)+f_{u}^{d}(y)\right)+u,
\end{gathered}
$$

whence III follows by I and 5.5 (vii).

LEMNA 8.7. Suppose that the binary operation + in $\Delta(\mathfrak{H})$ and the element $u \in A$ satisfy $\left(\mathrm{Z}_{2}\right),\left(\mathrm{Z}_{3}\right)$ and $\left(\mathrm{Z}_{4}\right)$. Then for all $f, g \in D F(\mathfrak{U})$ and $z \epsilon A$, the condition obtained from $\left(\mathrm{Z}_{4}\right)$ by replacing $u$ by $f_{u} g_{u} f^{d}(z)$ is also satisfied.

Proof. Assuming that

$$
f_{u} g_{u} f^{d}{ }_{u}(z)+x=f_{u} g_{u} f^{d}{ }_{u}(z)+y
$$

we add $f_{u} g^{d}{ }_{u} f_{u}^{d}(x)$ on the left and use 8.6 and 8.1 (ii) to obtain

$$
u+(u+x)=u+(u+y) \text {. }
$$

Hence the conclusion follows by $\left(\mathrm{Z}_{4}\right)$.

THEOREM 8.8. Suppose that the binary operation + in $\Delta(\mathfrak{A})$ and the element $u \in A$ satisfy $\left(\mathrm{Z}_{2}\right),\left(\mathrm{Z}_{3}\right)$ and $\left(\mathrm{Z}_{4}\right)$, and suppose there exist no elements $c, \bar{c} \epsilon A$ such that $c \neq u$ and $c+\bar{c}=u+u$, and such that the conditions obtained from $\left(\mathrm{Z}_{2}\right),\left(\mathrm{Z}_{3}\right)$ and $\left(\mathrm{Z}_{4}\right)$ by replacing $u$ by c are satisfied. Then $\mathfrak{A}$ Thas the strict refinement property.

Proof. For any $f, g \in D F(\mathfrak{A})$ and $z \in A$, if we let $c=f_{u} g_{u} f^{a}{ }_{u}(z)$ and $\bar{c}=f_{u} g_{u}^{d} f^{d}{ }_{u}(z)$, then $c+\bar{c}=u+u$ by 8.1 (ii), and it follows from 8.1 (vii), 8.6 and 8.7 that the conditions obtained from $\left(Z_{2}\right),\left(Z_{3}\right)$ and $\left(Z_{4}\right)$ by replacing $u$ by $c$ are satisfied. Hence $c=u$. According to 8.2 this implies 
that $(\mathfrak{U}, u)$ has the intermediate refinement property, and we conclude by 8.4 that $\mathfrak{U}$ has the strict refinement property.

If $\mathfrak{O}$ is an algebra with a zero element, then it is not hard to show that the mapping $E \rightarrow 0 / E$ is a one-to-one ccrrespondence between $F R(\mathfrak{A})$ and the set of all factor subalgebras of $\mathfrak{A}$, ard that for any factor relations $E$ and $F$ of $\mathfrak{U}$ the conditions $i d_{A}=E \times F$ and $A=(0 / E) \times(0 / F)$ are equivalent. Consequently certain results concerning inner direct prcducts of algebras can be refcrmulated in terms of factcr relations. As an example, Thecrem 3 in Fell-Tarski [5] yields the following results: An algebra $\mathfrak{A}$ with a zero element has the strict refinement property if and only if, for all $E, F, G \in F R(\mathfrak{U})$, the condition $i d_{A}=E \times F=E \times G$ implies that $F=G$. It is not known whether the assumption that $\mathfrak{A}$ has a zero element can be replaced by weaker conditions, for instance by some combination of the conditions $\left(Z_{1}\right)-\left(Z_{5}\right)$.

In conclusion we should like to make the following remark. In this paper we have obtained results concerning the refinement property by investigating two stronger conditions, the strong refinement property and the intermediate refinement property. In the case of algebras with a zero element similar results have been obtained by studying so-called exchange properties. ${ }^{5}$ ) There are of course cases where the refinement property holds although the stronger conditions fail, and for the investigation of such structures new methcds are needed. Just how much remains to be done in this area is indicated by the fact that it is not known whether every finite algebra $\langle A,+\rangle$ with a binary operation + that is commutative and associative has the unique factorization property.

\section{References}

[1] G. Birkhoff, Lattice theory, Rerised ed., Amer. Math. Soc. Coll. Publ., vol. 25, New York, 1948.

[2] C. C. Chang, Two theorems on direct decompositions of relations, Bull. Amer. Math. Soc. 60 (1954), p. 524.

[3] - Cardinal and ordinal multiplication of relation types, Proceedings of Symposia in Pure Mathematies, vol. 2, Lattice Theory, Amer. Math. Soc. 1961, pp. 123-128. [4] P. Crawley and B. Jónsson, Refinements for infinite direct decompositions of algebraic systems. To appear.

[5] J. M. G. Fell and A. Tarski, On algebras whose factor algebras are Boolean, Pac. J. of Math. 2 (1952), pp. 297-318.

[6] J. Hashimoto, Direct, subdireet decompositions and congruence relations, Osaka Math. J. 9 (1957), pp. 97-112.

(5) Strictly speaking, these investigations are concerned with a different operation, the so-called weak direct product, and it is only in the case of finitely many factors that two notions coincide. For a systematic study of exchange properties and their relations to the refinement property, see e.g. Crawley-Jónsson [4].
[7] B. Jónsson and A. Tarski, Decompositions on algebras, Bull. Amer. Math. Soc. 59 (1953), p. 77.

[8] - Direct decompositions of finite algebraic systems, Notre Dame Mathematical Lectures, No. 5 (1947).

[9] - Factor relations over algebras, Bull. Amer. Math. Soc. 59 (1953), p. 77.

[10] A. Tarski, Cardinal algebras, with an appendix by B. Jónsson and A. Tarski. Cardinal products of isomorphism types, New York, 1949.

UNIVERSITY OF CALIFORNIA AT LOS ANGELES

UNIVERSITY OF MINNESOTA, MINNEAPOLIS

Rẹu par la Rédaction le 20. 5. 1963 